\title{
CONSTRUCTION OF TWO-DIMENSIONAL QUANTUM FIELD MODELS THROUGH LONGO-WITTEN ENDOMORPHISMS
}

\author{
YOH TANIMOTO \\ Graduate School of Mathematical Sciences, The University of Tokyo, \\ Institut für Theoretische Physik, Universität Göttingen, \\ Friedrich-Hund-Platz 1, 37077 Göttingen, Germany; \\ email: yoh.tanimoto@theorie.physik.uni-goettingen.de
}

Received 1 September 2013; accepted 4 February 2014

\begin{abstract}
We present a procedure for constructing families of local, massive and interacting Haag-Kastler nets on the two-dimensional spacetime through an operator-algebraic method. A proof of existence of local observables is given without relying on modular nuclearity. By a similar technique, another family of wedge-local nets is constructed using certain endomorphisms of conformal nets recently studied by Longo and Witten.
\end{abstract}

2010 Mathematics Subject Classification: 81 T05 (primary), 81T40, 81 U99 (secondary)

\section{Introduction}

In a series of papers $[6,18,34]$ we have investigated operator-algebraic methods based on conformal field theory in order to construct quantum field models on two-dimensional spacetime with a weak localization property. Although we succeeded in obtaining various examples and general structural results, there was one important property missing: strict localization. Namely, we have constructed certain operator-algebraic objects which are considered to represent observables localized in the wedge-shaped regions in two-dimensional spacetime, but we could not prove the existence of observables in compactly localized regions. In the present paper we construct further new families of quantum field models and prove their strict locality. In other words, we construct interacting twodimensional Haag-Kastler nets, using techniques from conformal nets.

(c) The Author 2014. The online version of this article is published within an Open Access environment subject to the conditions of the Creative Commons Attribution licence <http://creativecommons.org/licenses/by/3.0/>. 
In recent years the operator-algebraic approach (algebraic QFT) to quantum field theory has seen many developments. A fundamental idea is that the whole quantum field model can be recovered from the set of observables localized in an unbounded wedge-shaped region and the spacetime translations [7]. Conversely, a strategy for constructing models is first to obtain models localized in wedges, then to prove the existence of fully localized observables. We say that they are wedge-local and strictly local, respectively. The most successful application of this strategy is the construction of scalar factorizing $S$-matrix models in two dimensions [22].

Construction of interacting quantum field theory in four dimensions remains one of the most important open problems in mathematical physics. Further steps toward higher dimensions within the above operator-algebraic approach have been obtained [13, 23]; however, the second step in finding localized observables has so far turned out to be unsuccessful [13]. Furthermore, we found that conformal covariance implies triviality of scattering in two dimensions [35], yet it is possible to construct weakly localized, conformally covariant models [34]. Thus the construction of wedge-local models should be considered as an important but intermediate step.

We have constructed weakly localized massless models using chiral components of two-dimensional conformal field theory [6, 18, 34]; however, strict locality is disproved in some of them and open in the others. The only known operator-algebraic technique for finding local observables is wedgesplitting [12] (see Section 4). This property is apparently difficult to have held in massless models, and is in particular known to be invalid in the conformal case. Actually, one encounters a similar problem in the form factor bootstrap program for integrable models [32]: one starts with a given $S$-matrix, and matrix coefficients of local operators are given as solutions of the so-called form factor equations. One can easily observe that the convergence of these matrix coefficients is much worse in the massless case. Hence, in order to avoid such technical difficulty, one should try to construct massive models (besides, the convergence of form factors is a difficult problem even for massive models [1]).

Although our previous constructions are fundamentally based on conformal field theory, one can still find a connection with massive models. We will investigate this issue more systematically in a separate paper [5]. Here we use again Longo-Witten endomorphisms [27] to construct weakly localized massive models. Then we show that some of them are actually strictly local by examining wedge-splitting. Note that in [22], wedge-splitting has been proved through modular nuclearity [12]. We provide both a direct proof of wedge-splitting and a simple proof of the modular nuclearity of certain models. Once strict locality is demonstrated, one can apply the standard scattering theory and check that the $S$-matrix is nontrivial. As we will see, this construction can be considered as a 
generalization of the Federbush model. The Federbush model is an integrable model with the $S$-matrix independent from the rapidity [29]. Local observables in this model have been found in [30] for certain range of coupling constant. We construct Haag-Kastler nets which have the same $S$-matrix as the Federbush models with the coupling constant of arbitrary size. The model describes multiple species of particles; hence the strict locality has not been treated in [22]. We construct another family of wedge-local nets (Borchers triples) using LongoWitten endomorphisms found in [27]. Although strict locality is open for this latter family, two constructions look quite similar.

The present construction uses as a starting point the free field or any quantum field which gives a wedge-split net. The new models are constructed on the tensor product of the Hilbert space of the input field (net). One could call our construction a 'deformation' of the trivial combination of the input fields. The term 'deformation' had a specific sense in [13, 23], and in massless cases it has been revealed that the connection with free (trivial scattering) models is generic [34]. In addition, one notes that such a 'deformation' is always related to a certain structure of the input field. The deformation in [13] exploits the translation covariance, while the chiral decomposition plays a crucial role in $[6,34]$. If one desires further different deformations, it is natural to introduce a further structure to the 'undeformed' model. In this paper this idea will be realized using a tensor product and inner symmetry. Such structure is easily found in examples. Indeed, our procedure in Section 3 can be applied to the tensor product of Lechner's models [22] and immediately gives new strict local nets.

This paper is organized as follows. After Sections 1 and 2, which are introductory, we first exhibit a way to construct Borchers triples. Section 3 is of general nature: given a Borchers triple with a certain symmetry, we show a way to construct new Borchers triples. Then we turn to strict locality in Section 4. We collect arguments for proving strict locality of the models from Section 3. This is our main result. Then we apply them to the concrete cases including the complex massive free field to obtain interacting Haag-Kastler nets (Section 5). Section 6 demonstrates how to construct massive Borchers triples from LongoWitten endomorphisms of the U(1)-current net. The two-particle $S$-matrix of these models will be also calculated. We do not investigate strict locality of these models.

\section{Preliminaries}

2.1. The two-dimensional Haag-Kastler net and Borchers triples. In algebraic QFT, models of quantum field theory are realized as nets of von Neumann algebras [20]. A Haag-Kastler net (of von Neumann algebras) $\mathcal{A}$ on 
$\mathbb{R}^{2}$ is a map $O \mapsto \mathcal{A}(O)$ from the set of open regions $\{O\}$ in two-dimensional Minkowski space $\mathbb{R}^{2}$ to the set of von Neumann algebras on a fixed Hilbert space $\mathcal{H}$ such that:

(1) (Isotony) If $O_{1} \subset O_{2}$, then $\mathcal{A}\left(O_{1}\right) \subset \mathcal{A}\left(O_{2}\right)$.

(2) (Locality) If $O_{1}$ and $O_{2}$ are spacelike separated, then $\mathcal{A}\left(O_{1}\right)$ and $\mathcal{A}\left(O_{2}\right)$ commute.

(3) (Poincaré covariance) There is a (strongly continuous) unitary representation $U$ of the (proper and orthochronous) Poincaré group $\mathcal{P}_{+}^{\uparrow}$ such that $U(g) \mathcal{A}(O) U(g)^{*}=\mathcal{A}(g O)$ for $g \in \mathcal{P}_{+}^{\uparrow}$.

(4) (Positivity of energy) The joint spectrum of the generators of the translation subgroup $\left(\cong \mathbb{R}^{2}\right)$ in the representation $U$ is contained in the closed forward lightcone $V_{+}:=\left\{\left(p_{0}, p_{1}\right) \in \mathbb{R}^{2}: p_{0} \geqslant\left|p_{1}\right|\right\}$.

(5) (Vacuum vector) There is an (up to scalar) unique vector $\Omega$ which is invariant under $U(g)$ and cyclic for $\mathcal{A}(O)$.

Note that the condition on the vacuum $\Omega$ contains the Reeh-Schlieder property, which can be derived if one assumes the additivity of the net. We take this as an axiom for the net for simplicity (see the discussion in [36, Section 2]). From these assumptions, the following is automatic [3]:

(6) (Irreducibility) The von Neumann algebra $\bigvee_{O \subset \mathbb{R}}{ }^{2} \mathcal{A}(O)$ is equal to $B(\mathcal{H})$.

To be precise, the triple $(\mathcal{A}, U, \Omega)$ should be called a Haag-Kastler net; however we say that $\mathcal{A}$ is a net if no confusion arises. If one has a net $\mathcal{A}$, under certain conditions one can define an $S$-matrix (see Section 5). The main objective in this paper is constructing examples of two-dimensional Haag-Kastler nets with nontrivial $S$-matrix.

However, it appears very difficult to construct such nets from a scratch. Fortunately, Borchers showed that it suffices to have a triple of a single von Neumann algebra associated with the wedge-shaped region $W_{\mathrm{R}}:=\left\{a \in \mathbb{R}^{2}\right.$ : $\left.a_{1}>\left|a_{0}\right|\right\}$, the spacetime translations and the vacuum. More precisely, a Borchers triple is a triple $(\mathcal{M}, T, \Omega)$ of a von Neumann algebra $\mathcal{M}$, a unitary representation $U$ of $\mathbb{R}^{2}$ and a vector $\Omega$ such that:

(1) If $a \in W_{\mathrm{R}}$, then $\operatorname{Ad} T(a)(\mathcal{M}) \subset \mathcal{M}$.

(2) The joint spectrum of the generators of $T$ is contained in $V_{+}$.

(3) $\Omega$ is cyclic and separating for $\mathcal{M}$. 
The correspondence between nets and Borchers triples is as follows (see [7, 22]). If $(\mathcal{A}, U, \Omega)$ is a (two-dimensional) Haag-Kastler net, then $\left(\mathcal{A}\left(W_{\mathrm{R}}\right),\left.U\right|_{\mathbb{R}^{2}}, \Omega\right)$ is a Borchers triple, where $U$ is restricted to the translation subgroup $\cong \mathbb{R}^{2}$.

On the other hand, if $(\mathcal{M}, T, \Omega)$ is a Borchers triple, then one can define a net as follows: in the two-dimensional Minkowski space any double cone $D$ is represented as the intersection of two wedges $D=\left(W_{\mathrm{R}}+a\right) \cap\left(W_{\mathrm{L}}+b\right)$, where $W_{\mathrm{L}}$ is the standard left wedge $W_{\mathrm{L}}:=\left\{a \in \mathbb{R}^{2}:-a_{1}>\left|a_{0}\right|\right\}$. Then we define for double cones $\mathcal{A}(D):=\operatorname{Ad} T(a)(\mathcal{M}) \cap \operatorname{Ad} T(b)\left(\mathcal{M}^{\prime}\right)$. For a general region $O$ we take $\mathcal{A}(O):=\bigvee_{D \subset O} \mathcal{A}(D)$, where the union runs over all the double cones contained in $O$. Then one can observe that $\mathcal{A}$ satisfies isotony and locality. Borchers further proved that the representation $T$ of $\mathbb{R}^{2}$ extends to a representation $U$ of $\mathcal{P}_{+}^{\uparrow}$ through Tomita-Takesaki theory [7] such that $\mathcal{A}$ is covariant and $\Omega$ is invariant under $U$ (the representation of boosts is given by the modular group). Positivity of energy is inherited from $T$ of the Borchers triple. The only missing property is cyclicity of $\Omega$ for local algebras $\mathcal{A}(O)$. Indeed, there are examples of Borchers triples which fail to satisfy this cyclicity [34, Theorem 4.16].

Hence, a general strategy for constructing Haag-Kastler nets is first to construct Borchers triples and then to check cyclicity of the vacuum. This program was first completed in [22]. Note that the latter condition is actually very hard to check directly. In [22], Lechner proved instead so-called modular nuclearity [12], which is a sufficient condition for the cyclicity of the vacuum. In this paper, we will provide new examples of Borchers triples for which the cyclicity of the vacuum can be proved without relying on modular nuclearity (see Section 4.2). We say that a Borchers triple is strictly local if $\Omega$ is cyclic for $\mathcal{A}(O)$ constructed as above. A strictly local Borchers triple corresponds to a Haag-Kastler net.

If there is a unitary operator $V$ which commutes with $U(g)$ and if $\operatorname{Ad} V$ preserves each local algebra $\mathcal{A}(O)$, then we call $\operatorname{Ad} V$ an inner symmetry of the net $\mathcal{A}$. By the uniqueness of $\Omega, \operatorname{Ad} V$ preserves the vacuum state $\langle\Omega, \cdot \Omega\rangle$. By the irreducibility, the automorphism Ad $V$ is implemented uniquely by $V$ up to a scalar. We require always that $V \Omega=\Omega$. By this requirement, the implementation is unique. A general strategy for using them in order to construct new Borchers triples will be explained in Section 3 and concrete examples of inner symmetry will be discussed in Section 5. A collection of inner symmetries may form a group $G$. In such a case we say that $G$ acts on the net by inner symmetry. Similarly one can consider an action of $V$ on Borchers triples. In this case, one says that $V$ implements an inner symmetry if $\operatorname{Ad} V$ preserves $\mathcal{M}$ and commutes with $T$. We say also that $G$ acts by inner symmetry when such operators $V$ form a group.

2.2. Conformal nets and Longo-Witten endomorphisms. In some of our constructions, the main ingredients come from chiral conformal field theory. Let 
us summarize here its operator-algebraic treatment (see also $[19,26])$. An open, connected, nondense and nonempty subset $I$ of $S^{1}$ is called an interval in $S^{1}$. We identify $\mathbb{R}$ as a dense subset in $S^{1}$ by the stereographic projection. The Möbius group PSL $(2, \mathbb{R})$ acts on $S^{1}=\mathbb{R} \cup\{\infty\}$ and under this identification it contains translations and dilations of $\mathbb{R}$. A (Möbius covariant) net (of von Neumann algebras) on $S^{1}$ is an assignment of von Neumann algebras $\mathcal{A}_{0}(I)$ on a common Hilbert space $\mathcal{H}_{0}$ to intervals $I$ such that:

(1) (Isotony) If $I_{1} \subset I_{2}$, then $\mathcal{A}_{0}\left(I_{1}\right) \subset \mathcal{A}_{0}\left(I_{2}\right)$.

(2) (Locality) If $I_{1}$ and $I_{2}$ are disjoint, then $\mathcal{A}_{0}\left(I_{1}\right)$ and $\mathcal{A}_{0}\left(I_{2}\right)$ commute.

(3) (Möbius covariance) There is a (strongly continuous) unitary representation $U_{0}$ of $\operatorname{PSL}(2, \mathbb{R})$ such that $U_{0}(g) \mathcal{A}_{0}(I) U_{0}(g)^{*}=\mathcal{A}_{0}(g I)$ for $g \in \operatorname{PSL}(2, \mathbb{R})$.

(4) (Positivity of energy) The generator of the subgroup of translation in the representation $U_{0}$ has positive spectrum.

(5) (Vacuum vector) There is an (up to scalar) unique vector $\Omega_{0}$ which is invariant under $U_{0}(g)$ and cyclic for $\mathcal{A}_{0}(I)$.

As in two dimensions, we call $\mathcal{A}_{0}$ a net; however the actual object of interest is the triple $\left(\mathcal{A}_{0}, U_{0}, \Omega_{0}\right)$. From these assumptions many properties automatically follow, among which the ones of importance in our application are:

(6) (Irreducibility) The von Neumann algebra $\bigvee_{I \subset S^{1}} \mathcal{A}_{0}(I)$ is equal to $B\left(\mathcal{H}_{0}\right)$.

(7) (Haag duality on $S^{1}$ ) It holds that $\mathcal{A}_{0}(I)^{\prime}=\mathcal{A}_{0}\left(I^{\prime}\right)$, where $I^{\prime}$ denotes the interior of the complement of $I$ in $S^{1}$.

(8) (The Bisognano-Wichmann property) The modular group $\Delta_{0}^{i t}$ of $\mathcal{A}_{0}\left(\mathbb{R}_{+}\right)$ associated with $\Omega_{0}$ is equal to $U_{0}(\delta(-2 \pi t))$, where $\delta$ is the dilation in $\operatorname{PSL}(2, \mathbb{R})$.

An inner symmetry of a conformal net $\mathcal{A}_{0}$ is a collection of automorphisms of each local algebra $\mathcal{A}_{0}(O)$ implemented by a common unitary operator $V_{0}$ which preserves the vacuum state $\left\langle\Omega_{0}, \cdot \Omega_{0}\right\rangle$. In the conformal (Möbius covariant) case, it automatically follows that $V_{0}$ commutes with $U_{0}(g)$ thanks to the BisognanoWichmann property.

A Longo-Witten endomorphism of $\mathcal{A}_{0}$ is an endomorphism of $\mathcal{A}_{0}\left(\mathbb{R}_{+}\right)$, implemented by a unitary operator $V_{0}$ which commutes with $U_{0}(g)$, where $g$ is a translation. An inner symmetry restricted to $\mathcal{A}_{0}\left(\mathbb{R}_{+}\right)$is a Longo-Witten endomorphism.

We will give a concrete example of a conformal net and of Longo-Witten endomorphisms in Section 6; these are not inner symmetries. Note that the term 
'Longo-Witten endomorphism' in this paper does not mean the concrete family found in [27]. In fact, in [27] it has been pointed out that for a Longo-Witten endomorphism of a conformal net there is a time-translation covariant net of von Neumann algebra on the half-plane in $\mathbb{R}^{2}$. A further family of examples has been found in [6].

2.3. The massive scalar free field. Our main construction strategy is based on simpler examples with certain properties. Let us quickly review the simplest quantum field.

Free fields are constructed from irreducible representations of the Poincaré group through second quantization. We use a notation which is to some extent consistent with that of [25]. The one-particle Hilbert space is $\mathcal{H}_{1}:=L^{2}(\mathbb{R}$, $d \theta$ ) and for the mass $m>0$ the (proper orthochronous) Poincare group acts through $U_{1}(a, \lambda) \psi(\theta)=e^{i p(\theta) \cdot a} \psi(\theta-\lambda)$, where $p(\theta):=(m \cosh (\theta), m \sinh (\theta))$ parameterizes the mass shell. We introduce the (auxiliary) unsymmetrized Hilbert space $\mathcal{H}^{\Sigma}:=\bigoplus \mathcal{H}_{1}^{\otimes n}$ and the (physical) symmetrized Hilbert space $\mathcal{H}_{\mathrm{r}}:=$ $\bigoplus Q_{n} \mathcal{H}_{1}^{\otimes n}$, where $Q_{n}$ is the projection onto the symmetric subspace.

Let us denote the $n$th component of a vector $\Psi \in \mathcal{H}_{\mathrm{r}}$ by $(\Psi)_{n}$. For a vector $\psi \in \mathcal{H}_{1}$ and $\Psi$ which has only finitely many components, the creation operator $b^{\dagger}$ is defined by $\left(b^{\dagger}(\psi) \Psi\right)_{n}=\sqrt{n} Q_{n}\left(\psi \otimes \Psi_{n-1}\right)$. The annihilation operator is the adjoint $b(\psi)=b^{\dagger}(\psi)^{*}$. With this notation, $b^{\dagger}$ is linear and $b$ is antilinear with respect to $\psi$. The free quantum field $\phi$ is now given by

$$
\phi(f):=b^{\dagger}\left(f^{+}\right)+b\left(J_{1} f^{-}\right), \quad f^{ \pm}(\theta)=\frac{1}{2 \pi} \int d^{2} a f(a) e^{ \pm i p(\theta) \cdot a},
$$

where $f$ is a test function in $\mathscr{S}\left(\mathbb{R}^{2}\right)$ and $J_{1} \psi(\theta)=\overline{\psi(\theta)}$. This field is local, in the sense that if $f$ and $g$ have spacelike separated supports, then $\phi(f)$ and $\phi(g)$ commute on an appropriate domain.

Finally we introduce the free field net. For each open region $O \subset \mathbb{R}^{2}$, we set

$$
\mathcal{A}_{\mathrm{r}}(O):=\left\{e^{i \phi(f)}: \operatorname{supp} f \subset O\right\}^{\prime \prime} .
$$

We have the second-quantized representation $U_{\mathrm{r}}:=\Gamma\left(U_{1}\right)$ and the Fock vacuum vector $\Omega_{\mathrm{r}} \in \mathcal{H}_{\mathrm{r}}$. This triple $\left(\mathcal{A}_{\mathrm{r}}, U_{\mathrm{r}}, \Omega_{\mathrm{r}}\right)$ is a two-dimensional Haag-Kastler net and referred to as the free massive scalar net. The subscript $r$ is to indicate the real scalar field. Furthermore, this net satisfies the modular nuclearity [12, Section 4], a property which we will explain in more detail in Section 4.

2.4. Scalar factorizing $S$-matrix models. The free net has many important features, but is not interacting. Lechner has constructed a large family of 
interacting nets [22]. Here we only briefly summarize the construction and their fundamental properties.

Let $S_{2}(\theta)$ be a bounded analytic function on the strip $0<\operatorname{Im} \theta<\pi$, continuous on the boundary, with the property

$$
S_{2}(\theta)^{-1}=\overline{S_{2}(\theta)}=S_{2}(-\theta)=S_{2}(\theta+i \pi)
$$

for $\theta \in \mathbb{R}$. This is called the two-particle scattering function.

This time again, the construction of the (wedge-local) field and the net is based on the one-particle space $\mathcal{H}_{1}$ above. There is a representation of the symmetric group $\mathfrak{S}_{n}$ on $\mathcal{H}_{1}^{\otimes n}$. For $\Psi_{n} \in \mathcal{H}_{1}^{\otimes n}$, the ' $S_{2}$-transposition' is given by

$$
\left(D_{S_{2}, n}\left(\tau_{j}\right) \Psi\right)\left(\theta_{1}, \ldots, \theta_{n}\right)=S_{2}\left(\theta_{j+1}-\theta_{j}\right) \Psi\left(\theta_{1}, \ldots, \theta_{j+1}, \theta_{j}, \ldots, \theta_{n}\right)
$$

for $\tau_{j}$ which transposes $j$ and $j+1$, and this generates a representation of $\mathfrak{S}_{n}$.

Let $Q_{S_{2}, n}$ be the orthogonal projection onto the subspace of $\mathcal{H}_{1}^{\otimes n}$ invariant under $\left\{D_{S_{2}, n}\left(\tau_{j}\right): 1 \leqslant j \leqslant n-1\right\}$. The full Hilbert space is $\mathcal{H}_{S_{2}}:=\bigoplus Q_{S_{2}, n} \mathcal{H}_{1}^{\otimes n}$ and the representation $U_{1}$ promotes to $U_{S_{2}}$ on $\mathcal{H}_{S_{2}}$ by second quantization. We define similarly the creation and annihilation operators $\left(z_{S_{2}}^{\dagger}(\psi) \Phi\right)_{n}=\sqrt{n} Q_{S_{2}, n}(\psi \otimes$ $\left.\Phi_{n-1}\right)$ and $z_{S_{2}}(\psi)=z_{S_{2}}^{\dagger}(\psi)^{*}$. The quantum field is defined accordingly by

$$
\phi_{S_{2}}(f):=z_{S_{2}}^{\dagger}\left(f^{+}\right)+z_{S_{2}}\left(J_{1} f^{-}\right), \quad f^{ \pm}(\theta)=\frac{1}{2 \pi} \int d^{2} a f(a) e^{ \pm i p(\theta) \cdot a},
$$

but this time $\phi_{S_{2}}$ is only wedge-local; hence the net is defined through Borchers triple.

The von Neumann algebra $\mathcal{M}_{S_{2}}$ is defined by

$$
\mathcal{M}_{S_{2}}:=\left\{e^{i \phi_{S_{2}}(f)}: \operatorname{supp} f \subset W_{\mathrm{L}}\right\}^{\prime}
$$

(note that here we take only the single commutant and $f$ has support in $W_{\mathrm{L}}$, while $\mathcal{M}_{S_{2}}$ corresponds to $W_{\mathrm{R}}$; by the wedge-duality, this is just a matter of convention (see [25])). The triple $\left(\mathcal{M}_{S_{2}}, U_{S_{2}}, \Omega_{S_{2}}\right)$ is a Borchers triple [21]. Furthermore, the modular nuclearity holds if $S_{2}$ fulfills a certain regularity condition and $S_{2}(0)=-1$ [22]; hence the triple is wedge-split in those cases.

\section{Borchers triples through inner symmetries}

In this section we make the first step in our main construction in two dimensions. We start with a Borchers triple with inner symmetry and construct new triples. Note that, differently from the case for our previous results $[6,34]$, the new triples are defined on a different Hilbert space although the formulas look very similar. 
First we treat the case where a given triple admits an action of $S^{1}$ by inner symmetry. Let us state a key lemma, which can be obtained as a special case of [34, Lemma 4.1].

LEMmA 3.1. Let $\mathcal{M}_{\mathrm{c}}$ be a von Neumann algebra and $Q_{\mathrm{c}}$ a self-adjoint operator such that $\operatorname{Ad} e^{i 2 \pi \kappa} Q_{\mathrm{c}}\left(\mathcal{M}_{\mathrm{c}}\right)=\mathcal{M}_{\mathrm{c}}$ for any $\kappa \in \mathbb{R}$. Then, for $\kappa \in \mathbb{R}, x \otimes \mathbb{1}$ commutes with $\operatorname{Ad} e^{i 2 \pi \kappa Q_{\mathrm{c}} \otimes Q_{\mathrm{c}}}\left(x^{\prime} \otimes \mathbb{1}\right)$ where $x \in \mathcal{M}_{\mathrm{c}}$ and $x^{\prime} \in \mathcal{M}_{\mathrm{c}}^{\prime}$.

Proof. For any $\kappa \in \mathbb{R}, x$ commutes with $\operatorname{Ad} e^{i 2 \pi \kappa Q_{\mathrm{c}}}\left(x^{\prime}\right)$ by assumption; hence the commutativity lemma [34, Lemma 4.1] applies.

Note that our assumption is for every $\kappa \in \mathbb{R}$, since in the partial spectral decomposition in the proof of [34, Lemma 4.1], there appears the action $\operatorname{Ad} e^{i 2 \pi q \kappa Q_{\mathrm{c}}}$ on the left component, $q \in \mathbb{R}$. Actually, if the spectrum of $Q_{\mathrm{c}}$ is contained in a subset $X \subset \mathbb{R}$, then it is enough to assume that $\operatorname{Ad} e^{i 2 \pi q \kappa} Q_{\mathrm{c}}\left(\mathcal{M}_{\mathrm{c}}^{\prime}\right) \subset$ $\mathcal{M}_{\mathrm{c}}^{\prime}$ for any $q \in X$. We will treat concrete cases where sp $Q_{\mathrm{c}} \subset \mathbb{Z}$. Here we put in the factor $2 \pi$ in order to keep the notation homogeneous with the actions of $\mathbb{Z}_{N}$ considered later.

Now let $\left(\mathcal{M}_{\mathrm{c}}, T_{\mathrm{c}}, \Omega_{\mathrm{c}}\right)$ be a Borchers triple with an action of $S^{1}=\mathbb{R} / \mathbb{Z}$ by inner symmetry. There is a unique unitary operator $V_{\mathrm{c}}(\kappa)$ which implements the inner symmetry through $S^{1}$. One takes the generator $Q_{\mathrm{c}}$ such that $V_{\mathrm{c}}(\kappa)=e^{i 2 \pi \kappa Q_{\mathrm{c}}}$. It is clear that $Q_{\mathrm{c}}$ commutes with the translation $T_{\mathrm{c}}$. One sees that $\operatorname{sp} Q_{\mathrm{c}} \subset \mathbb{Z}$ since $\mathbb{1}=V_{\mathrm{c}}(1)=e^{i 2 \pi Q_{\mathrm{c}}}$.

Now we turn to the construction of Borchers triples. Our objects act on the tensor product Hilbert space $\widetilde{\mathcal{H}}_{\mathrm{c}}:=\mathcal{H}_{\mathrm{c}} \otimes \mathcal{H}_{\mathrm{c}}$. Let us define $\widetilde{V}_{\mathrm{c}, \kappa}=e^{i 2 \pi \kappa Q_{\mathrm{c}} \otimes Q_{\mathrm{c}}}$. The tensor product representation $\widetilde{T}_{\mathrm{c}}(a):=T_{\mathrm{c}}(a) \otimes T_{\mathrm{c}}(a)$ has positive spectrum and preserves the new vacuum $\widetilde{\Omega}_{\mathrm{c}}:=\Omega_{\mathrm{c}} \otimes \Omega_{\mathrm{c}}$. The von Neumann algebra is given by

$$
\widetilde{\mathcal{M}}_{\mathrm{c}, \kappa}:=\left\{x \otimes \mathbb{1}, \text { Ad } \widetilde{V}_{\mathrm{c}, \kappa}(\mathbb{1} \otimes y): x, y \in \mathcal{M}_{\mathrm{c}}\right\}^{\prime \prime} .
$$

Note the difference from our previous construction [34]: first of all, this time the input is the two-dimensional Borchers triple, while we used chiral components in [34]. Accordingly, the representation $\widetilde{T}_{\mathrm{c}}$ is now just the two copies of the given one. The formula for $\widetilde{\mathcal{M}}_{\mathrm{c}, \kappa}$ is also similar, but this time we take both $x$ and $y$ from the same algebra $\mathcal{M}_{\mathrm{c}}$.

THEOREM 3.2. The triple $\left(\widetilde{\mathcal{M}}_{\mathrm{c}, \kappa}, \widetilde{T}_{\mathrm{c}}, \widetilde{\Omega}_{\mathrm{c}}\right)$ is a Borchers triple for each $\kappa \in \mathbb{R}$.

Proof. As in [34, Theorem 4.17], the properties of $\widetilde{T}_{\mathrm{c}}$ and $\widetilde{\Omega}_{\mathrm{c}}$ are readily checked. As for the relation $\operatorname{Ad}_{\widetilde{T}_{\mathrm{c}}}(a)\left(\widetilde{\mathcal{M}}_{\mathrm{c}, \kappa}\right) \subset \widetilde{\mathcal{M}}_{\mathrm{c}, \kappa}$ for $a \in W_{\mathrm{R}}$, one can prove this by noting that $\widetilde{V}_{\mathrm{c}, \kappa}$ and $\widetilde{T}_{\mathrm{c}}(a)$ commute and the assumptions that $\mathcal{M}_{\mathrm{c}}$ and $T_{\mathrm{c}}$ have the relation. 
The cyclicity of $\widetilde{\Omega}_{\mathrm{c}}$ for $\widetilde{\mathcal{M}}_{\mathrm{c}, \kappa}$ follows from the cyclicity of $\Omega_{\mathrm{c}}$ for $\mathcal{M}_{\mathrm{c}}$ and the fact that $\widetilde{V}_{\mathrm{c}, \kappa}(\mathbb{1} \otimes y) \widetilde{\Omega}_{\mathrm{c}}=(\mathbb{1} \otimes y) \widetilde{\Omega}_{\mathrm{c}}$. To show the separating property, we need again to prepare a sufficiently big algebra in the commutant:

$$
\widetilde{\mathcal{M}}_{\mathrm{c}, \kappa}^{1}:=\left\{\operatorname{Ad} \widetilde{V}_{\mathrm{c}, k}\left(x^{\prime} \otimes \mathbb{1}\right), \mathbb{1} \otimes y^{\prime}: x^{\prime}, y^{\prime} \in \mathcal{M}_{\mathrm{c}}^{\prime}\right\}^{\prime \prime} .
$$

It is easy to see that $\widetilde{\Omega}_{\mathrm{c}}$ is cyclic for $\widetilde{\mathcal{M}}_{\mathrm{c}, \kappa}^{1}$. In order to see that $\widetilde{\Omega}_{\mathrm{c}}$ is separating for $\widetilde{\mathcal{M}}_{\mathrm{c}, \kappa}$, it is enough to prove that $\widetilde{\mathcal{M}}_{\mathrm{c}, \kappa}$ and $\widetilde{\mathcal{M}}_{\mathrm{c}, \kappa}^{1}$ commute. This follows from Lemma 3.1, as in [34, Theorem 4.2].

A similar construction is possible if a Borchers triple admits an action of the finite group $\mathbb{Z}_{N}$ by inner symmetry. For a $\mathbb{Z}_{N}$-action we take $V_{\mathrm{c}}$ such that $V_{\mathrm{c}}^{k}$ implements the inner symmetry for $k \in \mathbb{Z}_{N}$. One can choose $Q_{\mathrm{c}}$ such that $V_{\mathrm{c}}=e^{i \frac{2 \pi}{N} Q_{\mathrm{c}}}$. We remark that we can concretely choose $Q_{\mathrm{c}}$ as follows: for each integer $j \in[0, N-1]$ we put $\hat{V}_{\mathrm{c}}(j):=\frac{1}{N} \sum_{k} e^{-i \frac{2 \pi j k}{N}} V_{\mathrm{c}}(k)$. It is clear that $\hat{V}_{\mathrm{c}}(j)$ is an orthogonal projection and $\hat{V}_{\mathrm{c}}(j) \hat{V}_{\mathrm{c}}(l)=0$ if $j \not \equiv l \bmod N$. In other words, $k \mapsto V_{\mathrm{c}}^{k}$ is a representation of $\mathbb{Z}_{N}$, whose dual group is also $\mathbb{Z}_{N}$, and we extract the spectral projections. Then we can take $Q_{\mathrm{c}}:=\sum_{j} j \hat{V}_{\mathrm{c}}(j)$. By this construction, it is clear that $Q_{\mathrm{c}}$ commutes with the translation $T_{\mathrm{c}}$. An operator $Q_{\mathrm{c}}$ with the properties specified above is not unique since one can amplify each spectral component by $N$, but if we consider the operator $e^{i \frac{2 \pi k}{N} Q_{\mathrm{c}} \otimes Q_{\mathrm{c}}}$, it does not depend on the choice of $Q_{\mathrm{c}}$ (see also the remark after [34, Theorem 4.17]). One sees that $\operatorname{sp} Q_{\mathrm{c}} \subset \mathbb{Z}$ since $\mathbb{1}=V_{\mathrm{c}}(1)=e^{i 2 \pi Q_{\mathrm{c}}}$ for an $S^{1}$-action or $\mathbb{1}=V_{\mathrm{c}}^{N}=e^{i 2 \pi Q_{\mathrm{c}}}$ for a $\mathbb{Z}_{N}$-action (or directly by our choice).

Analogously to Lemma 3.1, one can prove the following. Here, the assumption is for all integers and so is the result.

LEMMA 3.3. Let $N$ be an integer, $\mathcal{M}_{\mathrm{c}}$ a von Neumann algebra and $Q_{\mathrm{c}}$ a selfadjoint operator such that $\operatorname{sp} Q_{\mathrm{c}} \subset \mathbb{Z}$ and $\operatorname{Ad} e^{i \frac{2 \pi k}{N} Q_{\mathrm{c}}}\left(\mathcal{M}_{\mathrm{c}}\right)=\mathcal{M}_{\mathrm{c}}$ for any $k \in \mathbb{Z}$. Then, for $k \in \mathbb{Z}, x \otimes \mathbb{1}$ commutes with $\operatorname{Ad} e^{i \frac{2 \pi k}{N} Q_{\mathrm{c}} \otimes Q_{\mathrm{c}}}\left(x^{\prime} \otimes \mathbb{1}\right)$ where $x \in \mathcal{M}_{\mathrm{c}}$ and $x^{\prime} \in \mathcal{M}_{\mathrm{c}}^{\prime}$.

The construction of Borchers triples is also parallel. We take the tensor product Hilbert space $\widetilde{\mathcal{H}}_{\mathrm{c}}:=\mathcal{H}_{\mathrm{c}} \otimes \mathcal{H}_{\mathrm{c}}$, the tensor product representation $\widetilde{T}_{\mathrm{c}}(a):=T_{\mathrm{c}}(a) \otimes$ $T_{\mathrm{c}}(a)$ and the vacuum $\widetilde{\Omega}_{\mathrm{c}}:=\Omega_{\mathrm{c}} \otimes \Omega_{\mathrm{c}}$. We define $\widetilde{V}_{\mathrm{c}, k}=e^{i \frac{2 \pi k}{N} Q_{\mathrm{c}} \otimes Q_{\mathrm{c}}}$ and

$$
\widetilde{\mathcal{M}}_{\mathrm{c}, k}:=\left\{x \otimes \mathbb{1}, \text { Ad } \widetilde{V}_{\mathrm{c}, k}(\mathbb{1} \otimes y): x, y \in \mathcal{M}_{\mathrm{c}}\right\}^{\prime \prime} .
$$

THEOREM 3.4. The triple $\left(\widetilde{\mathcal{M}}_{\mathrm{c}, k}, \widetilde{T}_{\mathrm{c}}, \widetilde{\Omega}_{\mathrm{c}}\right)$ is a Borchers triple for each $k \in \mathbb{Z}_{N}$.

At the end of this section, we remark that the existence of inner symmetry is not at all exceptional. Indeed, if $\left(\mathcal{M}_{\mathrm{c}}, T_{\mathrm{c}}, \Omega_{\mathrm{c}}\right)$ is a (wedge-split) Borchers triple, then 
the tensor product $\left(\mathcal{M}_{\mathrm{c}} \otimes \mathcal{M}_{\mathrm{c}}, T_{\mathrm{c}} \otimes T_{\mathrm{c}}, \Omega_{\mathrm{c}} \otimes \Omega_{\mathrm{c}}\right)$ has the flip automorphism which commutes with $T_{\mathrm{c}} \otimes T_{\mathrm{c}}$ and preserves $\Omega_{\mathrm{c}} \otimes \Omega_{\mathrm{c}}$; hence it is an inner symmetry $\mathbb{Z}_{2}$. We present more examples in Section 5.

\section{General arguments for strict locality}

We are now concerned with the main problem in the construction of nets through Borchers triples: the strict locality of the models in Section 3. The key argument is the wedge-split property.

In general, if one has an inclusion of von Neumann algebras $\mathcal{N} \subset \mathcal{M}$, this is said to be split if there is a type I factor $\mathcal{R}$ such that $\mathcal{N} \subset \mathcal{R} \subset \mathcal{M}$. Furthermore, if $\mathcal{N} \subset \mathcal{M}$ is an inclusion of factors and $\Omega$ is cyclic and separating for $\mathcal{M}$, then the nuclearity of the map $\mathcal{N} \ni x \longmapsto \Delta^{\frac{1}{4}} x \Omega$ implies the split property, where $\Delta$ is the modular operator for $\mathcal{M}$ with respect to $\Omega$ [11, Proposition 2.3].

We say that a Borchers triple $(\mathcal{M}, T, \Omega)$ is wedge-split if $\operatorname{Ad} T(a)(\mathcal{M}) \subset \mathcal{M}$ is split for any $a \in W_{\mathrm{R}}$. Note that $W_{\mathrm{R}}$ is defined as the open wedge, and split inclusion for lightlike translation is not required. The wedge-split property implies that the inclusion $\operatorname{Ad} T(a)(\mathcal{M}) \subset \mathcal{M}$ is unitarily equivalent to $\left(\mathcal{M}_{2} \otimes \mathbb{C} \mathbb{1}\right) \subset$ $\left(B\left(\mathcal{K}_{1}\right) \otimes \mathcal{M}_{1}\right)$, where $\mathcal{M}_{1}$ and $\mathcal{M}_{2}$ cannot be trivial since $\mathcal{M}$ is of type III; hence the intersection $\mathcal{M} \cap \operatorname{Ad} T(a)(\mathcal{M})^{\prime}$ for $a \in W_{\mathrm{R}}$ is unitarily equivalent to $\mathcal{M}_{2}^{\prime} \otimes \mathcal{M}_{1}$ which is nontrivial [12]. Let $\Delta$ be the modular operator for $\mathcal{M}$ with respect to $\Omega$. One says that an inclusion $\mathcal{N} \subset \mathcal{M}$ satisfies modular nuclearity if the map $\mathcal{N} \ni x \longmapsto \Delta^{\frac{1}{4}} x \Omega$ is nuclear. A Borchers triple $(\mathcal{M}, T, \Omega)$ is said to satisfy modular nuclearity if the inclusion $\operatorname{Ad} T(\mathcal{M}) \subset \mathcal{M}$ has modular nuclearity for any $a \in W_{\mathrm{R}}$. From the above remark, modular nuclearity implies the wedge-split property. The strict locality of the models in [22] was proved through modular nuclearity.

Actually, wedge-split inclusion is sufficient for the strict locality [22, Theorem 2.5].

THEOREM 4.1 (Lechner). If a Borchers triple $(\mathcal{M}, T, \Omega)$ is wedge-split, then it is strictly local.

This theorem is stated with the assumption of modular nuclearity; however, the actual proof depends only on the wedge-split property.

Let us recall our main construction strategy (Section 3): starting with a given Borchers triple $\left(\mathcal{M}_{\mathrm{c}}, T_{\mathrm{c}}, \Omega_{\mathrm{c}}\right)$ with inner symmetry Ad $V_{\mathrm{c}}(\kappa)$ of $S^{1}$, we constructed a new Borchers triple $\left(\widetilde{\mathcal{M}}_{\mathrm{c}, \kappa}, \widetilde{T}_{\mathrm{c}}, \widetilde{\Omega}_{\mathrm{c}}\right)$ on the tensor product Hilbert space. As we will see later (Section 5), this gives a nontrivial scattering even if the initial triple comes from the free field. This can be generalized to the following program: take 
a Borchers triple with a good property (either the wedge-split property or modular nuclearity) and prove that the construction of Section 3 leads again to Borchers triples with the same property.

We carry out this program in two ways. First we present a proof through the wedge-split property and then we use modular nuclearity. Note that strict locality is not 'good enough' for this program. We will exhibit examples of strictly local Borchers triples for which the new triples constructed as in Section 3 violate strict locality. Another remark is that our arguments in Section 4.2 through the wedgesplit property are valid for both $S^{1}$ - and $\mathbb{Z}_{N}$-actions, but those in Section 4.3 through modular nuclearity apply so far only to finite cyclic group actions. Already for the simplest compact group $S^{1}$ the proofs break down, as we will see.

4.1. Common arguments. Let us collect some facts which will be frequently used in the following (see also [34, Section 4.4.1]). In this subsection, we do not use the properties of the Borchers triple.

In the following, we consider only an action of $S^{1}$. The case of $\mathbb{Z}_{N}$ is analogous. Let $\mathcal{M}_{\mathrm{c}}$ be a von Neumann algebra, $\Omega_{\mathrm{c}}$ be a cyclic separating vector for $\mathcal{M}_{\mathrm{c}}$ and $V_{\mathrm{c}}(\kappa)$ be a unitary representation of $S^{1}$ which preserves $\Omega_{\mathrm{c}}$ such that $\alpha_{\mathrm{c}, \kappa}:=$ $\operatorname{Ad} V_{\mathrm{c}}(\kappa)$ is an automorphism of $\mathcal{M}_{\mathrm{c}}$ and $V_{\mathrm{c}}(1)=\mathbb{1}$. In other words, there is an action $\alpha_{\mathrm{c}}$ of $S^{1}$ on $\mathcal{M}_{\mathrm{c}}$ which preserves the state $\left\langle\Omega_{\mathrm{c}}, \cdot \Omega_{\mathrm{c}}\right\rangle$.

Discrete Fourier expansion on von Neumann algebras. For any element $x \in \mathcal{M}_{\mathrm{c}}$ and $l \in \mathbb{Z}$, we define

$$
x_{l}:=\int_{0}^{1} d \kappa \operatorname{Ad} V_{\mathrm{c}}(\kappa)(x) e^{-i 2 \pi l \kappa} .
$$

It holds that $x=\sum_{l \in \mathbb{Z}} x_{l}$ and $\operatorname{Ad} V_{\mathrm{c}}(\kappa)\left(x_{l}\right)=\alpha_{\mathrm{c}, \kappa}\left(x_{l}\right)=e^{i 2 \pi l \kappa} x_{l}$. The sum is *-strongly convergent. Let us denote by $\mathcal{M}_{\mathrm{c}}^{\alpha_{\mathrm{c}}}(l)$ the set of elements $x$ of $\mathcal{M}_{\mathrm{c}}$ such that $\alpha_{\mathrm{c}, \kappa}(x)=e^{i 2 \pi l \kappa} x$. Then it holds that $x_{l} \in \mathcal{M}_{\mathrm{c}}^{\alpha_{\mathrm{c}}}(l)$ and $\mathcal{M}_{\mathrm{c}}^{\alpha_{\mathrm{c}}}\left(l_{1}\right) \cdot \mathcal{M}_{\mathrm{c}}^{\alpha_{\mathrm{c}}}\left(l_{2}\right) \subset$ $\mathcal{M}_{\mathrm{c}}^{\alpha_{\mathrm{c}}}\left(l_{1}+l_{2}\right)$.

These are well-known facts; however, if necessary the reader is referred to [34, Proposition 4.9] for a proof (for the case of $\mathbb{Z}_{N}$, the proof is easy to adapt).

Next we consider the twisting. As in Section 3, let $Q_{\mathrm{c}}$ be a self-adjoint operator such that $V_{\mathrm{c}}(\kappa)=e^{i 2 \pi \kappa Q_{\mathrm{c}}}$. Then for $y \in \mathcal{M}_{\mathrm{c}}^{\alpha_{\mathrm{c}}}(m)$, it holds that

$$
\operatorname{Ad} \widetilde{V}_{\mathrm{c}, \kappa}(\mathbb{1} \otimes y)=\operatorname{Ad} e^{i 2 \pi \kappa Q_{\mathrm{c}} \otimes Q_{\mathrm{c}}}(\mathbb{1} \otimes y)=e^{i 2 \pi m \kappa Q_{\mathrm{c}}} \otimes y=V_{\mathrm{c}}(m \kappa) \otimes y,
$$

where $\widetilde{V}_{\mathrm{c}, \kappa}=e^{i 2 \pi \kappa Q_{\mathrm{c}} \otimes Q_{\mathrm{c}}}$ as in Section 3. For the proof, see [34, Lemma 4.10]. The proof is written again for an action of $S^{1}$; however, the adaptation is easy for $\mathbb{Z}_{N}$. Note that one has to consider spectral subspaces of $V_{\mathrm{c}}$ parameterized by $\mathbb{Z}_{N}$. 
The identification $\mathbb{Z}_{N}=\mathbb{Z} / N \mathbb{Z}$ should always be kept in mind and numbers, for example, $e^{i \frac{2 \pi k}{N}}$, are well-defined for $k \in \mathbb{Z}_{N}$.

Finally we remark that $\widetilde{\mathcal{M}}_{\mathrm{c}, \kappa}$ is the $*$-strong closure of the linear span of the elements of the form $x_{l} V_{\mathrm{c}}(m \kappa) \otimes y_{m}$, where $x_{l} \in \mathcal{M}_{\mathrm{c}}^{\alpha_{\mathrm{c}}}(l)$ and $y_{m} \in \mathcal{M}_{\mathrm{c}}^{\alpha_{\mathrm{c}}}(m)$. Furthermore, any element $\tilde{x} \in \widetilde{\mathcal{M}}_{\mathrm{c}, \kappa}$ can be decomposed as follows:

$$
\tilde{x}=\sum_{l, m \in \mathbb{Z}} \tilde{x}_{l, m}\left(V_{\mathrm{c}}(m \kappa) \otimes \mathbb{1}\right), \quad \tilde{x}_{l, m} \in \mathcal{M}_{\mathrm{c}} \otimes \mathcal{M}_{\mathrm{c}}
$$

and the sum is $*$-strongly convergent. This decomposition corresponds to the discrete Fourier expansion with respect to the action $\left(\operatorname{Ad} V_{c}\right) \otimes\left(\operatorname{Ad} V_{c}\right)$ of the group $S^{1} \times S^{1}$. The group $S^{1} \times S^{1}$ acts also on $\mathcal{M}_{\mathrm{c}} \otimes \mathcal{M}_{\mathrm{c}}$ and there is a decomposition into components as well. For a corresponding proof, see again [34, Lemma 4.14].

The modular operator and inner symmetry. Let $\Delta_{\mathrm{c}}$ and $J_{\mathrm{c}}$ be the modular operator and the modular conjugation for $\mathcal{M}_{\mathrm{c}}$ with respect to $\Omega_{\mathrm{c}}$. The implementing unitary $V_{\mathrm{c}}$ of $\alpha_{\mathrm{c}}$ and $\Delta_{\mathrm{c}}, J_{\mathrm{c}}$ commute. The modular operator and the modular conjugation for $\mathcal{M}_{\mathrm{c}} \otimes \mathcal{M}_{\mathrm{c}}$ with respect to $\widetilde{\Omega}_{\mathrm{c}}:=\Omega_{\mathrm{c}} \otimes \Omega_{\mathrm{c}}$ are $\Delta_{\mathrm{c}} \otimes \Delta_{\mathrm{c}}$ and $J_{\mathrm{c}} \otimes J_{\mathrm{c}}$, respectively. Now we can determine the modular objects for $\widetilde{\mathcal{M}}_{\mathrm{c}, \kappa}=\mathcal{M}_{\mathrm{c}} \otimes \mathbb{1} \vee \operatorname{Ad} \widetilde{V}_{\mathrm{c}, \kappa}\left(\mathbb{1} \otimes \mathcal{M}_{\mathrm{c}}\right)$.

PROPOSITION 4.2. The operator $\Delta_{\mathrm{c}} \otimes \Delta_{\mathrm{c}}$ is the modular operator for $\widetilde{\mathcal{M}}_{\mathrm{c}, k}$ with respect to $\widetilde{\Omega}_{\mathrm{c}}$.

Proof. We will give two proofs. The first one is a direct calculation and the second one uses the so-called KMS condition.

A direct calculation goes as follows. We will see that actually the modular conjugation is $\widetilde{V}_{\mathrm{c}, \kappa}\left(J_{\mathrm{c}} \otimes J_{\mathrm{c}}\right)$. Since we have already the candidates for the modular objects, we only have to check their actions. First we take $x_{l} \in \mathcal{M}_{\mathrm{c}}^{\alpha_{\mathrm{c}}}(l)$ and $y_{m} \in$ $\mathcal{M}_{\mathrm{c}}^{\alpha_{\mathrm{c}}}(m)$. The operator $x_{l} V_{\mathrm{c}}(m \kappa) \otimes y_{m}$ belongs to $\widetilde{\mathcal{M}}_{\mathrm{c}, \kappa}$ and it holds that

$$
\begin{aligned}
\widetilde{V}_{\mathrm{c}, \kappa}\left(J_{\mathrm{c}} \otimes J_{\mathrm{c}}\right)\left(\Delta_{\mathrm{c}} \otimes \Delta_{\mathrm{c}}\right)\left(x_{l} V_{\mathrm{c}}(m \kappa) \otimes y_{m}\right) \widetilde{\Omega}_{\mathrm{c}} & =\widetilde{V}_{\mathrm{c}, \kappa}\left(S_{c} \otimes S_{c}\right)\left(x_{l} \Omega_{\mathrm{c}} \otimes y_{m} \Omega_{\mathrm{c}}\right) \\
& =\widetilde{V}_{\mathrm{c}, \kappa}\left(x_{l}^{*} \Omega_{\mathrm{c}} \otimes y_{m}^{*} \Omega_{\mathrm{c}}\right) \\
& =e^{i 2 \pi l m \kappa}\left(x_{l}^{*} \Omega_{\mathrm{c}} \otimes y_{m}^{*} \Omega_{\mathrm{c}}\right) \\
& =\left(\alpha_{\mathrm{c},-m \kappa}\left(x_{l}^{*}\right) \Omega_{\mathrm{c}}\right) \otimes\left(y_{m}^{*} \Omega_{\mathrm{c}}\right) \\
& =\left(V_{\mathrm{c}}(-m \kappa) x_{l}^{*} \Omega_{\mathrm{c}}\right) \otimes\left(y_{m}^{*} \Omega_{\mathrm{c}}\right) \\
& =\left(x_{l} V_{\mathrm{c}}(m \kappa) \otimes y_{m}\right)^{*}\left(\Omega_{\mathrm{c}} \otimes \Omega_{\mathrm{c}}\right),
\end{aligned}
$$

namely, the operator $\widetilde{V}_{\mathrm{c}, \kappa}\left(J_{\mathrm{c}} \otimes J_{\mathrm{c}}\right)\left(\Delta_{\mathrm{c}} \otimes \Delta_{\mathrm{c}}\right)$ acts correctly as the modular involution. Since $\widetilde{\mathcal{M}}_{\mathrm{c}, \kappa}$ is the $*$-strong closure of the linear span of such elements, 
the modular involution actually coincides with $\widetilde{V}_{\mathrm{c}, \kappa}\left(J_{\mathrm{c}} \otimes J_{\mathrm{c}}\right)\left(\Delta_{\mathrm{c}} \otimes \Delta_{\mathrm{c}}\right)$. Then the conclusion follows immediately from the uniqueness of the polar decomposition.

In the second proof, we use the uniqueness of the modular automorphism group with respect to the KMS condition. In general, for a von Neumann algebra $\mathcal{M}$ and a state $\psi$, if there is a one-parameter automorphism $\sigma_{t}$ which satisfies the KMS condition, namely if for any pair $x, y \in \mathcal{M}$ there is an analytic function $f(t)$ on the strip $0<\operatorname{Im} t<1$ and continuous on the boundary such that $f(t)=\psi\left(\sigma_{t}(x) y\right)$ and $f(t+i)=\psi\left(y \sigma_{t}(x)\right)$ for $t \in \mathbb{R}$, then $\sigma_{t}$ is the modular automorphism [33, Theorem VIII.1.2]. In our case, the state is the vacuum $\left\langle\widetilde{\Omega}_{\mathrm{c}}, \cdot \widetilde{\Omega}_{\mathrm{c}}\right\rangle$. This is the KMS state on the von Neumann algebra $\mathcal{M}_{\mathrm{c}} \otimes \mathcal{M}_{\mathrm{c}}$ with respect to $\sigma_{t}^{\widetilde{\Omega}_{\mathrm{c}}}=\operatorname{Ad}\left(\Delta_{\mathrm{c}}^{i t} \otimes \Delta_{\mathrm{c}}^{i t}\right)$. Hence for any pair $\tilde{x}, \tilde{y} \in \mathcal{M}_{\mathrm{c}} \otimes \mathcal{M}_{\mathrm{c}}$ of elements there is an analytic function as above. Actually the decomposition with respect to $\alpha_{\mathrm{c}} \otimes \alpha_{\mathrm{c}}$ commutes with $\sigma^{\widetilde{\Omega}_{\mathrm{c}}}$ (see the remark about discrete Fourier expansion) and it holds that

$$
\begin{aligned}
& \left\langle\widetilde{\Omega}_{\mathrm{c}}, \sigma_{t}^{\widetilde{\Omega}_{\mathrm{c}}}(\tilde{x}) \tilde{y} \widetilde{\Omega}_{\mathrm{c}}\right\rangle=\sum_{l, m}\left\langle\widetilde{\Omega}_{\mathrm{c}}, \sigma_{t}^{\widetilde{\Omega}_{\mathrm{c}}}\left(\tilde{x}_{l, m}\right) \tilde{y}_{-l,-m} \widetilde{\Omega}_{\mathrm{c}}\right\rangle, \\
& \left\langle\widetilde{\Omega}_{\mathrm{c}}, \tilde{y} \sigma_{t}^{\widetilde{\Omega}_{\mathrm{c}}}(\tilde{x}) \widetilde{\Omega}_{\mathrm{c}}\right\rangle=\sum_{l, m}\left\langle\widetilde{\Omega}_{\mathrm{c}}, \tilde{y}_{-l,-m} \sigma_{t}^{\widetilde{\Omega}_{\mathrm{c}}}\left(\tilde{x}_{l, m}\right) \widetilde{\Omega}_{\mathrm{c}}\right\rangle
\end{aligned}
$$

by the orthogonality of the vacuum acted on by the component $\tilde{x}_{l, m}$ and so on. Next, by considering the pair $\tilde{x}_{l, m}, \tilde{y}_{-l,-m}$ and the KMS condition for $\mathcal{M}_{\mathrm{c}} \otimes \mathcal{M}_{\mathrm{c}}$, there is an analytic function

$$
\tilde{f}_{l, m}(t)=\left\langle\widetilde{\Omega}_{\mathrm{c}}, \sigma_{t}^{\widetilde{\Omega}_{\mathrm{c}}}\left(\tilde{x}_{l, m}\right) \tilde{y}_{-l,-m} \widetilde{\Omega}_{\mathrm{c}}\right\rangle, \tilde{f}_{l, m}(t+i)=\left\langle\widetilde{\Omega}_{\mathrm{c}}, \tilde{y}_{-l,-m} \sigma_{t}^{\widetilde{\Omega}_{\mathrm{c}}}\left(\widetilde{x}_{l, m}\right) \widetilde{\Omega}_{\mathrm{c}}\right\rangle
$$

(of course the dependence of $\widetilde{f}_{l, m}$ on $\tilde{x}$ and $\tilde{y}$ is implicit). Let us turn to $\widetilde{\mathcal{M}}_{\mathrm{c}, \kappa}$. By the same argument as above, an inner product decomposes into a suitable combination and the decomposition is compatible with the action of $\sigma_{t}^{\widetilde{\Omega}_{\mathrm{c}}}=$ $\operatorname{Ad}\left(\Delta_{\mathrm{c}}^{i t} \otimes \Delta_{\mathrm{c}}^{i t}\right)$. For a pair of elements $\tilde{x}_{l, m}\left(e^{i 2 \pi m \kappa} Q_{\mathrm{c}} \otimes \mathbb{1}\right)$ and $\tilde{y}_{-l,-m}\left(e^{-i 2 \pi m \kappa Q_{\mathrm{c}}} \otimes \mathbb{1}\right)$, where $\tilde{x}_{l, m}, \tilde{y}_{-l-m} \in \mathcal{M}_{\mathrm{c}} \otimes \mathcal{M}_{\mathrm{c}}$, we have

$$
\begin{aligned}
& \left\langle\widetilde{\Omega}_{\mathrm{c}}, \sigma_{t}^{\widetilde{\Omega}_{\mathrm{c}}}\left(\widetilde{x}_{l, m}\left(e^{i 2 \pi m \kappa} Q_{\mathrm{c}} \otimes \mathbb{1}\right)\right) \widetilde{y}_{-l,-m}\left(e^{-i 2 \pi m \kappa} Q_{\mathrm{c}} \otimes \mathbb{1}\right) \widetilde{\Omega}_{\mathrm{c}}\right\rangle \\
& \quad=e^{-i 2 \pi l m \kappa}\left\langle\widetilde{\Omega}_{\mathrm{c}}, \sigma_{t} \widetilde{\Omega}_{\mathrm{c}}\left(\widetilde{x}_{l, m}\right) \widetilde{y}_{-l,-m} \widetilde{\Omega}_{\mathrm{c}}\right\rangle, \\
& \left\langle\widetilde{\Omega}_{\mathrm{c}}, \tilde{y}_{-l,-m}\left(e^{-i 2 \pi m \kappa Q_{\mathrm{c}}} \otimes \mathbb{1}\right) \sigma_{t}^{\widetilde{\Omega}_{\mathrm{c}}}\left(\widetilde{x}_{l, m}\left(e^{i 2 \pi m \kappa Q_{\mathrm{c}}} \otimes \mathbb{1}\right)\right) \widetilde{\Omega}_{\mathrm{c}}\right\rangle \\
& \quad=e^{-i 2 \pi l m \kappa}\left\langle\widetilde{\Omega}_{\mathrm{c}}, \tilde{y}_{-l,-m} \sigma_{t}^{\widetilde{\Omega}_{\mathrm{c}}}\left(\widetilde{x}_{l, m}\right) \widetilde{\Omega}_{\mathrm{c}}\right\rangle .
\end{aligned}
$$

One observes that the right hand sides are equal to $\widetilde{f}_{l, m}$ up to the constant $e^{-i 2 \pi l m \kappa}$. In other words, the KMS condition is satisfied for the pair with respect to $\sigma^{\widetilde{\Omega}_{c}}$. Therefore for an arbitrary linear combination of such components the 
KMS condition holds as well, thanks to the decomposition of the inner product into $(l, m)$-components. Such linear combinations are $*$-strongly dense in $\widetilde{\mathcal{M}}_{\mathrm{c}, \kappa}$; hence the above-cited uniqueness theorem applies, allowing one to see that $\sigma_{t}^{\widetilde{\Omega}_{\mathrm{c}}}=\operatorname{Ad}\left(\Delta_{\mathrm{c}}^{i t} \otimes \Delta_{\mathrm{c}}^{i t}\right)$ is the modular automorphism of $\widetilde{\mathcal{M}}_{\mathrm{c}, \kappa}$ with respect to $\left\langle\widetilde{\Omega}_{\mathrm{c}}, \cdot \widetilde{\Omega}_{\mathrm{c}}\right\rangle$ (indeed, the KMS condition on a $*$-strongly dense subalgebra is enough by [9, Proposition 5.7]. One should note that in [9] the KMS condition is defined on a dense set of analytic elements). Since $\Delta_{\mathrm{c}} \otimes \Delta_{\mathrm{c}}$ preserves the vacuum vector $\Omega_{\mathrm{c}} \otimes \Omega_{\mathrm{c}}$, it must coincide with the modular operator.

It is interesting to compare our proof with that of $[12,22]$ where the modular objects were calculated through unbounded operators affiliated to the von Neumann algebras. One recalls also that in our previous work the modular objects were indirectly determined by scattering theory [34, Section 3].

Proposition 4.3. It holds that $\left(\widetilde{\mathcal{M}}_{\mathrm{c}, k}\right)^{\prime}=\left(\operatorname{Ad} \widetilde{V}_{\mathrm{c}, k}\left(\mathcal{M}_{\mathrm{c}}^{\prime} \otimes \mathbb{1}\right) \vee \mathbb{1} \otimes \mathcal{M}_{\mathrm{c}}^{\prime}\right)=$ : $\widetilde{\mathcal{M}}_{\mathrm{c}, \kappa}^{1}$.

Proof. It can be observed that the right hand side commutes with $\widetilde{\mathcal{M}}_{\mathrm{c}, \kappa}$ by the same argument as in Theorem 3.2, so we have $\widetilde{\mathcal{M}}_{\mathrm{c}, \kappa}^{1} \subset\left(\widetilde{\mathcal{M}}_{\mathrm{c}, \kappa}\right)^{\prime}$. Since we already know that the modular operator for $\left(\widetilde{\mathcal{M}}_{\mathrm{c}, \kappa}\right)^{\prime}$ with respect to $\Omega_{\mathrm{c}} \otimes \Omega_{\mathrm{c}}$ is $\Delta_{\mathrm{c}}^{-1} \otimes \Delta_{\mathrm{c}}^{-1}$ from Proposition 4.2, it is immediately seen that $\widetilde{\mathcal{M}}_{\mathrm{c}, \kappa}^{1}$ is globally invariant under the modular group of $\left(\widetilde{\mathcal{M}}_{\mathrm{c}, \kappa}\right)^{\prime}$ with respect to $\Omega_{\mathrm{c}} \otimes \Omega_{\mathrm{c}}$. Then the two algebras coincide since $\Omega_{\mathrm{c}} \otimes \Omega_{\mathrm{c}}$ is cyclic for $\widetilde{\mathcal{M}}_{\mathrm{c}, \kappa}^{1}$ by a standard application of Takesaki's theorem [33, Theorem IX.4.2] (see [35, Theorem A.1]).

Variations of Propositions 4.2 and 4.3 hold for an action of $\mathbb{Z}_{N}$ with trivial changes.

4.2. Proof through the wedge-split property. Here we take as the starting point a Borchers triple $\left(\mathcal{M}_{\mathrm{c}}, T_{\mathrm{c}}, \Omega_{\mathrm{c}}\right)$ which is wedge-split. As before, we assume that there is an action of $S^{1}$ implemented by $V_{\mathrm{c}}(\kappa)$. With this action and for $\kappa \in \mathbb{R}$ we can construct a Borchers triple $\left(\widetilde{\mathcal{M}}_{\mathrm{c}, \kappa}, \widetilde{T}_{\mathrm{c}}, \widetilde{\Omega}_{\mathrm{c}}\right)$ as in Section 3 . We are going to prove that this is again wedge-split, and hence strictly local.

Recall that [15] for a split inclusion $\left(\mathcal{N}_{\mathrm{c}} \subset \mathcal{M}_{\mathrm{c}}, \Omega_{\mathrm{c}}\right)$ equipped with a cyclic separating vector $\Omega_{\mathrm{c}}$ for $\mathcal{M}_{\mathrm{c}}, \mathcal{N}_{\mathrm{c}}$ and $\mathcal{M}_{\mathrm{c}} \cap \mathcal{N}_{\mathrm{c}}^{\prime}\left(\right.$ where $\mathcal{N}_{\mathrm{c}}$ will be $\operatorname{Ad} T_{\mathrm{c}}(a)\left(\mathcal{M}_{\mathrm{c}}\right)$ for some $a \in W_{\mathrm{R}}$ ), there is a canonical type I factor $\mathcal{R}_{\mathrm{c}}$ such that $\mathcal{N}_{\mathrm{c}} \subset \mathcal{R}_{\mathrm{c}} \subset \mathcal{M}_{\mathrm{c}}$. Moreover, $\mathcal{R}_{\mathrm{c}}$ is given by the formula $\mathcal{R}_{\mathrm{c}}=\mathcal{N}_{\mathrm{c}} \vee J \mathcal{N}_{\mathrm{c}} J=\mathcal{M}_{\mathrm{c}} \cap J \mathcal{M}_{\mathrm{c}} J$, where $J$ is the modular conjugation for $\mathcal{M}_{\mathrm{c}} \cap \mathcal{N}_{\mathrm{c}}^{\prime}$ with respect to $\Omega_{\mathrm{c}}$. If $V_{\mathrm{c}}$ is a unitary operator which preserves $\Omega_{\mathrm{c}}$ and $\operatorname{Ad} V_{\mathrm{c}}$ preserves both $\mathcal{N}_{\mathrm{c}}$ and $\mathcal{M}_{\mathrm{c}}$, then $\mathcal{R}_{\mathrm{c}}$ is preserved under $\operatorname{Ad} V_{\mathrm{c}}$ as well. 
LEMMA 4.4. The von Neumann algebra $\widetilde{\mathcal{R}}_{\mathrm{c}, \kappa}:=\mathcal{R}_{\mathrm{c}} \otimes \mathbb{1} \vee \operatorname{Ad} \widetilde{V}_{\mathrm{c}, \kappa}\left(\mathbb{1} \otimes \mathcal{R}_{\mathrm{c}}\right)$ is a factor.

Proof. Since we know that $\left(\widetilde{\mathcal{R}}_{\mathrm{c}, \kappa}\right)^{\prime}=\operatorname{Ad} \widetilde{V}_{\mathrm{c}, \kappa}\left(\mathcal{R}_{\mathrm{c}}^{\prime} \otimes \mathbb{1}\right) \vee \mathbb{1} \otimes \mathcal{R}_{\mathrm{c}}^{\prime}$ by Proposition 4.3 (in Section 4.1 we did not assume that the von Neumann algebra comes from a Borchers triple), we only have to show that $\widetilde{\mathcal{R}}_{\mathrm{c}, \kappa} \vee\left(\widetilde{\mathcal{R}}_{\mathrm{c}, k}\right)^{\prime}=\mathcal{R}_{\mathrm{c}} \otimes \mathcal{R}_{\mathrm{c}}^{\prime} \vee$ Ad $\widetilde{V}_{\mathrm{c}, \kappa}\left(\mathcal{R}_{\mathrm{c}}^{\prime} \otimes \mathcal{R}_{\mathrm{c}}\right)$ is equal to $B\left(\mathcal{H}_{\mathrm{c}} \otimes \mathcal{H}_{\mathrm{c}}\right)$.

We show that $V_{\mathrm{c}}(\kappa) \otimes \mathbb{1}$ and $\mathbb{1} \otimes V_{\mathrm{c}}(\kappa)$ are contained in $\widetilde{\mathcal{R}}_{\mathrm{c}, \kappa} \vee\left(\widetilde{\mathcal{R}}_{\mathrm{c}, \kappa}\right)^{\prime}$ for each $\kappa \in \mathbb{R}$. Indeed, $V_{\mathrm{c}}(\kappa) \otimes \mathbb{1}$ implements an automorphism on $\mathcal{R}_{\mathrm{c}} \otimes \mathbb{1}$ and since the latter is a type I factor, there is a unitary $u \in \mathcal{R}_{\mathrm{c}}$ which implements the same automorphism. At the same time $V_{\mathrm{c}}(\kappa) \otimes \mathbb{1}$ implements an automorphism of $\mathcal{R}_{\mathrm{c}}^{\prime} \otimes \mathbb{1}$, and there is an implementing unitary $u^{\prime} \in \mathcal{R}_{\mathrm{c}}^{\prime}$. Then $u u^{\prime}$ implements Ad $V_{\mathrm{c}}(\kappa)$ on $\mathcal{B}\left(\mathcal{H}_{\mathrm{c}}\right)=\mathcal{R}_{\mathrm{c}} \vee \mathcal{R}_{\mathrm{c}}^{\prime}$; hence $u u^{\prime} V_{\mathrm{c}}(\kappa)^{*}$ must be a scalar. We may assume $u u^{\prime}=V_{\mathrm{c}}(\kappa)$. Furthermore, obviously $\operatorname{Ad} V_{\mathrm{c}}(\kappa)\left(u^{\prime}\right)=\operatorname{Ad} u^{\prime}\left(u^{\prime}\right)=u^{\prime}$, namely $u^{\prime}$ is fixed under the automorphism $\operatorname{Ad} V_{\mathrm{c}}(\kappa)$; hence $\operatorname{Ad} \widetilde{V}_{\mathrm{c}, \kappa}\left(u^{\prime} \otimes \mathbb{1}\right)=u^{\prime} \otimes \mathbb{1}$. This implies that $u^{\prime} \otimes \mathbb{1} \in\left(\widetilde{\mathcal{R}}_{\mathrm{c}, \kappa}\right)^{\prime}$ and hence $u u^{\prime} \otimes \mathbb{1}=V_{\mathrm{c}}(\kappa) \otimes \mathbb{1}$ is in $\widetilde{\mathcal{R}}_{\mathrm{c}, \kappa} \vee\left(\tilde{\widetilde{\mathcal{R}}}_{\mathrm{c}, \kappa}\right)^{\prime}$. An analogous proof works for $\mathbb{1} \otimes V_{\mathrm{c}}(\kappa)$.

The rest is easy since $\widetilde{V}_{\mathrm{c}, \kappa}$ is obtained from the functional calculus of $V_{\mathrm{c}}(\kappa) \otimes \mathbb{1}$ and $\mathbb{1} \otimes V_{\mathrm{c}}(\kappa)$.

This lemma actually works even for an action of $\mathbb{R}$. In contrast, we need the periodicity of the action in the following.

THEOREM 4.5. The von Neumann algebra $\widetilde{\mathcal{R}}_{\mathrm{c}, \kappa}:=\mathcal{R}_{\mathrm{c}} \otimes \mathbb{1} \vee \operatorname{Ad} \widetilde{V}_{\mathrm{c}, \kappa}\left(\mathbb{1} \otimes \mathcal{R}_{\mathrm{c}}\right)$ is a type I factor.

Proof. We have seen that $\widetilde{\mathcal{R}}_{\mathrm{c}, \kappa}$ is a factor. What remains is to show that $\widetilde{\mathcal{R}}_{\mathrm{c}, \kappa}$ contains a minimal projection. As in Lemma 4.4 we take the implementing unitary $u(\kappa) \in \mathcal{R}_{\mathrm{c}}$ for $\operatorname{Ad} V_{\mathrm{c}}(\kappa)$, this time indicating the dependence on $\kappa$. By a classical result by Bargmann, we may assume that $u(\kappa)$ is a one-parameter group of unitaries in $\mathcal{R}_{\mathrm{c}}$ [2, Theorem 1.1, Lemma 4.3], where our group is $S^{1}$ and hence a one-parameter group.

Thus the spectrum of the representation $u$ is discrete and contained in $\mathbb{Z}$ (by multiplying a one-parameter phase), and hence the fixed point $\mathcal{R}_{\mathrm{c}}^{\alpha_{\mathrm{c}}}=\mathcal{R}_{\mathrm{c}} \cap\{u(\kappa)$ : $\kappa \in \mathbb{R}\}^{\prime}$ is the relative commutant in $\mathcal{R}_{\mathrm{c}}$ of the spectral projections of the representation $u$. Hence it is the direct sum of type I factors and admits a minimal projection.

Let us take such a minimal projection $p$ in $\mathcal{R}_{\mathrm{c}}^{\alpha_{\mathrm{c}}}$. In general, if $y \in \mathcal{R}_{\mathrm{c}}^{\alpha_{\mathrm{c}}}$, then Ad $\widetilde{V}_{\mathrm{c}, \kappa}(\mathbb{1} \otimes y)=\mathbb{1} \otimes y$ and hence the inclusion $\mathcal{R}_{\mathrm{c}} \otimes \mathcal{R}_{\mathrm{c}}^{\alpha_{\mathrm{c}}} \subset \widetilde{\mathcal{R}}_{\mathrm{c}, \kappa}$ holds. Obviously $p$ must be a subprojection of a spectral projector of $u$ corresponding to a discrete 
eigenvalue. Furthermore, it is immediate that $p \otimes p$ is a minimal projection in $\mathcal{R}_{\mathrm{c}} \otimes \mathcal{R}_{\mathrm{c}}^{\alpha_{\mathrm{c}}}$. We claim that it is a minimal projection in $\widetilde{\mathcal{R}}_{\mathrm{c}, \kappa}$. In fact, suppose that $\widetilde{q} \leqslant p \otimes p$ in $\widetilde{\mathcal{R}}_{\mathrm{c}, \kappa}$. Then $\widetilde{q}$ and $\mathbb{1} \otimes u(\kappa)$ commute since the spectral projections of the representation $\mathbb{1} \otimes u$ either contain or are disjoint from $p \otimes p$; hence this also holds with $\widetilde{q}$. This implies that $\widetilde{q}$ belongs to $\mathcal{R}_{\mathrm{c}} \otimes \mathcal{R}_{\mathrm{c}}^{\alpha_{\mathrm{c}}}$ (consider the discrete Fourier expansion $\widetilde{q}=\sum \widetilde{q}_{l m}\left(V_{\mathrm{c}}(m \kappa) \otimes \mathbb{1}\right)$; then it is possible that $\operatorname{Ad}(1 \otimes u(\kappa))(\widetilde{q})=\widetilde{q}$ only if $\widetilde{q}_{l m}$ vanishes except for $\left.m=0\right)$; then $\widetilde{q}=p \otimes p$ by the minimality of $p \otimes p$ in this restricted algebra. This is the minimality of $p \otimes p$ in $\widetilde{\mathcal{R}}_{\mathrm{c}, \kappa}$.

We remark that the intermediate type I factor constructed here is not the canonical one of [15]. An explicit formula for it involves the modular conjugation of the relative commutant, which is only abstractly determined.

The proof of Theorem 4.5 can be easily adapted to the case of an action of $\mathbb{Z}_{N}$.

In terms of a wedge-split Borchers triple, we apply Theorem 4.5 with $\mathcal{N}_{\mathrm{c}}=$ $\operatorname{Ad} T_{\mathrm{c}}(a)\left(\mathcal{M}_{\mathrm{c}}\right)$ to see the split inclusion

$$
\begin{aligned}
\mathcal{N}_{\mathrm{c}} \otimes \mathbb{1} \vee \operatorname{Ad} \widetilde{V}_{\mathrm{c}, \kappa}\left(\mathbb{1} \otimes \mathcal{N}_{\mathrm{c}}\right) & \subset \mathcal{R}_{\mathrm{c}} \otimes \mathbb{1} \vee \operatorname{Ad} \widetilde{V}_{\mathrm{c}, \kappa}\left(\mathbb{1} \otimes \mathcal{R}_{\mathrm{c}}\right) \\
& \subset \mathcal{M}_{\mathrm{c}} \otimes \mathbb{1} \vee \operatorname{Ad} \widetilde{V}_{\mathrm{c}, \kappa}\left(\mathbb{1} \otimes \mathcal{M}_{\mathrm{c}}\right),
\end{aligned}
$$

where the middle element is $\widetilde{\mathcal{R}}_{\mathrm{c}, \kappa}$ and the last element is $\widetilde{\mathcal{M}}_{\mathrm{c}, \kappa}$. Since $\widetilde{T}_{\mathrm{c}}$ and $\widetilde{V}_{\mathrm{c}, \kappa}$ commute, Ad $\widetilde{T}_{\mathrm{c}}(a)\left(\widetilde{\mathcal{M}}_{\mathrm{c}, \kappa}\right) \subset \widetilde{\mathcal{M}}_{\mathrm{c}, \kappa}$ is split with an intermediate type I factor $\widetilde{\mathcal{R}}_{\mathrm{c}, \kappa}$ (which implicitly depends on $a$ ). Then we have the following with the help of Theorem 4.1.

THEOREM 4.6. If a given Borchers triple $\left(\mathcal{M}_{\mathrm{c}}, T_{\mathrm{c}}, \Omega_{\mathrm{c}}\right)$ with an action of $S^{1}$ by inner symmetry is wedge-split, then the Borchers triple $\left(\widetilde{\mathcal{M}}_{\mathrm{c}, \kappa}, \widetilde{T}_{\mathrm{c}}, \widetilde{\Omega}_{\mathrm{c}}\right)$ is wedgesplit, and hence strictly local.

One can repeat parallel proofs for an action of $\mathbb{Z}_{N}$ to obtain the same result.

4.3. Proof through modular nuclearity. Let us give another proof of strict locality, based on modular nuclearity. Here we have to restrict ourselves to the case of an action of $\mathbb{Z}_{N}$. Of course one can take an arbitrary finite subgroup $\mathbb{Z}_{N}$ in $S^{1}$; hence physically this should not be considered as an essential restriction. Let $\left(\widetilde{\mathcal{M}}_{\mathrm{c}, k}, \widetilde{T}_{\mathrm{c}}, \widetilde{\Omega}_{\mathrm{c}}\right)$ again be constructed as in Section 3 for a fixed $k \in \mathbb{Z}_{N}$. Again, thanks to Theorem 4.1, it is enough to prove the modular nuclearity of the new triple.

Let us start with a trivial observation.

LEMMA 4.7. Let $\left(\mathcal{M}_{\mathrm{c}}, T_{\mathrm{c}}, \Omega_{\mathrm{c}}\right)$ be a Borchers triple with modular nuclearity. Then the triple $\left(\mathcal{M}_{\mathrm{c}} \otimes \mathcal{M}_{\mathrm{c}}, \widetilde{T}_{\mathrm{c}}, \widetilde{\Omega}_{\mathrm{c}}\right)$ has modular nuclearity. 
Proof. The modular objects are the tensor products as well; hence the nuclearity norm simply gets squared.

THEOREM 4.8. Let $\left(\mathcal{M}_{\mathrm{c}}, T_{\mathrm{c}}, \Omega_{\mathrm{c}}\right)$ be a Borchers triple with modular nuclearity. Then the triple $\left(\widetilde{\mathcal{M}}_{\mathrm{c}, k}, \widetilde{T}_{\mathrm{c}}, \widetilde{\Omega}_{\mathrm{c}}\right)$ has modular nuclearity.

Proof. For a fixed $a \in W_{\mathrm{R}}$, we have to show that the inclusion $\operatorname{Ad} \widetilde{T}_{\mathrm{c}}(a)\left(\widetilde{\mathcal{M}}_{\mathrm{c}, k}\right) \subset$ $\widetilde{\mathcal{M}}_{\mathrm{c}, k}$ has modular nuclearity with respect to $\widetilde{\Omega}_{\mathrm{c}}$. By the assumption and Lemma 4.7 we know that $\left(\operatorname{Ad} \widetilde{T}_{\mathrm{c}}(a)\left(\mathcal{M}_{\mathrm{c}} \otimes \mathcal{M}_{\mathrm{c}}\right) \subset \mathcal{M}_{\mathrm{c}} \otimes \mathcal{M}_{\mathrm{c}}\right)$ has modular nuclearity. This means that the map

$$
\mathcal{M}_{\mathrm{c}} \otimes \mathcal{M}_{\mathrm{c}} \ni \tilde{x} \longmapsto\left(\Delta_{\mathrm{c}}^{\frac{1}{4}} \otimes \Delta_{\mathrm{c}}^{\frac{1}{4}}\right) \tilde{x}\left(\Omega_{\mathrm{c}} \otimes \Omega_{\mathrm{c}}\right)
$$

is a nuclear map.

Let us consider an element $\tilde{x}$ of $\mathcal{M}_{\mathrm{c}} \otimes \mathcal{M}_{\mathrm{c}}$. As we have seen at the end of Section 4.1 (before Proposition 4.3), it can be decomposed as $\tilde{x}=\sum_{l, m} \tilde{x}_{l, m}$, and $\tilde{x}_{l, m}$ are the matrix components with respect to the grading given by the $\mathbb{Z}_{N}$-action and each $\widetilde{x}_{l, m}$ is in $\mathcal{M}_{\mathrm{c}} \otimes \mathcal{M}_{\mathrm{c}}$. For such $\tilde{x}$, we define an element $\tau_{k}(\widetilde{x}) \in \widetilde{\mathcal{M}}_{\mathrm{c}, k}$ as follows:

$$
\tau_{k}(\tilde{x})=\sum_{l, m} \tilde{x}_{l, m}\left(V_{\mathrm{c}}^{k m} \otimes \mathbb{1}\right)
$$

This is a finite sum, and thus there is no problem of convergence. This map $\tau_{k}$ is onto, as any element in $\widetilde{\mathcal{M}}_{\mathrm{c}, k}$ in decomposed in the form above. It is important to observe that the action on the vector $\widetilde{\Omega}_{\mathrm{c}}$ is unchanged. In other words, it holds that $\tilde{x} \widetilde{\Omega}_{\mathrm{c}}=\tau_{k}(\tilde{x}) \widetilde{\Omega}_{\mathrm{c}}$.

Now, we know that the modular operator of $\widetilde{\mathcal{M}}_{\mathrm{c}, k}$ with respect to $\widetilde{\Omega}_{\mathrm{c}}$ is $\Delta_{\mathrm{c}} \otimes \Delta_{\mathrm{c}}$; hence the map in question is $\tau_{k}(\widetilde{x}) \longmapsto\left(\Delta_{\mathrm{c}}^{\frac{1}{4}} \otimes \Delta_{\mathrm{c}}^{\frac{1}{4}}\right) \tau_{k}(\widetilde{x}) \widetilde{\Omega}_{\mathrm{c}}=\left(\Delta_{\mathrm{c}}^{\frac{1}{4}} \otimes \Delta_{\mathrm{c}}^{\frac{1}{4}}\right) \widetilde{x} \widetilde{\Omega}_{\mathrm{c}}$. By the assumption and Lemma 4.7 we know that the map $\tilde{x} \longmapsto\left(\Delta_{\mathrm{c}}^{\frac{1}{4}} \otimes \Delta_{\mathrm{c}}^{\frac{1}{4}}\right) \tau_{k}(\tilde{x}) \widetilde{\Omega}_{\mathrm{c}}$ is nuclear.

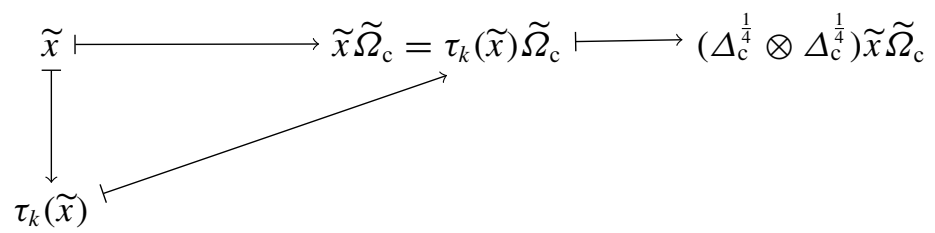

In the diagram above, the left triangle commutes and the straight line above is nuclear. Since a composition of a bounded linear map and a nuclear map is again nuclear, we only have to show that $\tau_{k}^{-1}$ is bounded. For this purpose, let us recall how explicitly $\left\{\widetilde{x}_{l, m}\right\}$ are obtained (see [34, Proposition 4.9] for a corresponding 
formula for $S^{1}$-action):

$$
\tilde{x}_{l, m}=\frac{1}{N^{2}} \sum_{j_{1}, j_{2}} e^{-i \frac{2 \pi\left(j_{1} l+j_{2} m\right)}{N}} \operatorname{Ad}\left(V_{\mathrm{c}}^{j_{1}} \otimes V_{\mathrm{c}}^{j_{2}}\right)(\widetilde{x}) .
$$

Correspondingly for the decomposition of $\tau_{k}(\tilde{x})$, we have

$$
\tilde{x}_{l, m}\left(V_{\mathrm{c}}^{k m} \otimes \mathbb{1}\right)=\frac{1}{N^{2}} \sum_{j_{1}, j_{2}} e^{-i \frac{2 \pi\left(j_{1} l+j_{2} m\right)}{N}} \operatorname{Ad}\left(V_{\mathrm{c}}^{j_{1}} \otimes V_{\mathrm{c}}^{j_{2}}\right)\left(\tau_{k}(\tilde{x})\right) .
$$

In particular, we see that $\left\|\tilde{x}_{l, m}\right\| \leqslant\left\|\tau_{k}(\tilde{x})\right\|$ and hence $\|\tilde{x}\|=\left\|\sum_{l, m} \tilde{x}_{l, m}\right\| \leqslant$ $N^{2}\left\|\tau_{k}(\widetilde{x})\right\|$. In other words, $\tau_{k}^{-1}$ is bounded by $N^{2}$.

Note that here the finiteness of $N$ is crucial. The author does not know whether the same holds for the action of for example $S^{1}$, although one can always take an arbitrary finite subgroup $\mathbb{Z}_{N}$ of $S^{1}$.

4.4. Counterexamples from the massless case. In previous sections we started with a strictly local Borchers triple, constructed new triples and proved strict locality. Although our method may seem quite simple, it is neither trivial nor purely group-theoretic. In order to see this, we take the massless models, for which one can construct Borchers triples but strict locality fails. See also [34, Section 4.4].

Let $\left(\mathcal{A}_{0}, T_{0}, \Omega_{0}\right)$ be any strongly additive conformal (diffeomorphism covariant) net with an action of $\mathbb{Z}_{N}$ by inner symmetry (there are many such nets, for example the tensor product of the U(1)-current or the loop group nets). A two-dimensional massless net can be constructed through a tensor product: $\mathcal{A}\left(I_{+} \times I_{-}\right):=\mathcal{A}_{0}\left(I_{+}\right) \otimes \mathcal{A}_{0}\left(I_{-}\right), T\left(t_{+}, t_{-}\right):=T_{0}\left(t_{+}\right) \otimes T_{0}\left(t_{-}\right), \Omega:=\Omega_{0} \otimes \Omega_{0}$. On the net $\mathcal{A}$ there is an action of $\mathbb{Z}_{N}$ by inner symmetry which acts only on the left component of $\mathcal{A}_{0}\left(I_{+}\right) \otimes \mathcal{A}_{0}\left(I_{-}\right)$. Let $Q$ be the generator with sp $Q \subset \mathbb{Z}$, which is of the form $Q_{0} \otimes \mathbb{1}$. With the wedge algebra $\mathcal{M}:=\mathcal{A}_{0}\left(\mathbb{R}_{-}\right) \otimes \mathcal{A}_{0}\left(\mathbb{R}_{+}\right)$, the twisted Borchers triple is given by

$$
\begin{aligned}
\widetilde{\mathcal{M}}_{\mathrm{c}, k} & :=\mathcal{M} \otimes \mathbb{1} \vee \operatorname{Ad} e^{i \frac{2 \pi k}{N} Q \otimes Q}(\mathbb{1} \otimes \mathcal{M}) \\
& =\mathcal{A}_{0}\left(\mathbb{R}_{-}\right) \otimes \mathcal{A}_{0}\left(\mathbb{R}_{+}\right) \otimes \mathbb{1} \vee \operatorname{Ad} e^{i \frac{2 \pi k}{N} Q \otimes Q}(\mathbb{1} \otimes \mathcal{M}) \\
& \cong\left(\mathcal{A}_{0}\left(\mathbb{R}_{-}\right) \otimes \mathbb{1} \vee \operatorname{Ad} e^{i \frac{2 \pi k}{N} Q_{0} \otimes Q_{0}}\left(\mathbb{1} \otimes \mathcal{A}_{0}\left(\mathbb{R}_{-}\right)\right)\right) \\
& \otimes\left(\mathcal{A}_{0}\left(\mathbb{R}_{+}\right) \otimes \mathcal{A}_{0}\left(\mathbb{R}_{+}\right)\right),
\end{aligned}
$$

where in the last line we interchanged the second and the third components for brevity. We consider the relative commutant of the wedge shifted by $a=\left(a_{+}, a_{-}\right)$, $a_{+}<0, a_{-}>0$. With the above interchanged notation, it is clear that the --component (the third and fourth tensor components) of the intersection is 
simply $\mathcal{A}_{0}\left(\left(0, a_{-}\right)\right) \otimes \mathcal{A}_{0}\left(\left(0, a_{-}\right)\right)$. As for the + -component (the first and second tensor components), one observes that it is almost same as the intersection calculated in [34, Theorem 4.16]. The only change is that the direction of the second component is reversed. This does not affect the proof; indeed, the inner symmetry commutes with translation and the positivity of energy is used only through the Reeh-Schlieder property and hence is not essential. Thus we have

$$
\begin{aligned}
\widetilde{\mathcal{M}}_{\mathrm{c}, k} \cap \operatorname{Ad} \widetilde{T}_{\mathrm{c}}(a)\left(\widetilde{\mathcal{M}}_{\mathrm{c}, k}\right)= & \left(\mathcal{A}_{0}^{\alpha_{0}}\left(\left(a_{+}, 0\right)\right) \otimes \mathcal{A}_{0}^{\alpha_{0}}\left(\left(a_{+}, 0\right)\right)\right) \\
& \otimes\left(\mathcal{A}_{0}\left(\left(0, a_{-}\right)\right) \otimes \mathcal{A}_{0}\left(\left(0, a_{-}\right)\right)\right),
\end{aligned}
$$

where $\mathcal{A}_{0}^{\alpha_{0}}$ denotes the fixed point with respect to $\operatorname{Ad} e^{i \frac{2 \pi k}{N}} Q_{0}$. In particular with $k=1$, this does not satisfy the Reeh-Schlieder property and strict locality fails if $\mathbb{Z}_{N}$ acts nontrivially.

This counterexample shows that our proof of locality is by no means purely group-theoretic. Namely, in order to obtain strict locality, it is necessary to assume a stronger property than strict locality itself of the original net (the wedge-split property or modular nuclearity as above). The above massless counterexample appears to be related to the subtlety in the massless bootstrap program: the convergence of form factors is typically less good in massless models and even the fundamental commutativity theorem relies on the behavior of form factors, which is also worse (see [32]). Hence if one aims at constructing Wightman fields or a Haag-Kastler net from form factors, the problem of convergence is inevitable.

\section{Realization as deformed fields and scattering theory}

In Section 3, we constructed families of Borchers triples by an operatoralgebraic method. Such a construction was also useful for the argument of strict locality for one case (Section 4). However, we still have to show that the resulting nets of von Neumann algebras are really new, or more desirably have nontrivial interaction, and for this purpose the previous presentation is not very convenient. Fortunately, it turns out that the nets are accompanied by (wedgelocal) quantum fields which create one-particle states and the scattering process can be calculated [8]. We follow the notation of [25].

The Zamolodchikov-Fadeev algebra. Here we consider the construction in Section 3 applied to the complex free massive field net. The complex massive free net is given simply by the tensor product $\mathcal{M}_{\mathrm{c}}:=\mathcal{M}_{\mathrm{r}} \otimes \mathcal{M}_{\mathrm{r}}, T_{\mathrm{c}}:=T_{\mathrm{r}} \otimes T_{\mathrm{r}}$, $\Omega_{\mathrm{c}}:=\Omega_{\mathrm{r}} \otimes \Omega_{\mathrm{r}}$. The Hilbert space $\mathcal{H}_{\mathrm{c}}:=\mathcal{H}_{\mathrm{r}} \otimes \mathcal{H}_{\mathrm{r}}$ is canonically isomorphic to the Fock space $\mathcal{F}\left(\mathcal{H}_{1} \oplus \mathcal{H}_{1}\right)$. The $\mathrm{U}(1)$ symmetry transformation of the complex field is constructed as follows. On the 'one-particle space' $\mathcal{H}_{1} \oplus \mathcal{H}_{1}$ we consider 
the following operator:

$$
V_{1}(\kappa)\left(\begin{array}{l}
\xi \\
\eta
\end{array}\right)=\left(\begin{array}{cc}
\cos 2 \pi \kappa & -\sin 2 \pi \kappa \\
\sin 2 \pi \kappa & \cos 2 \pi \kappa
\end{array}\right)\left(\begin{array}{l}
\xi \\
\eta
\end{array}\right), \quad \kappa \in \mathbb{R} .
$$

The second-quantized promotion to $\mathcal{H}_{\mathrm{c}}$ is denoted by $V_{\mathrm{c}}(\kappa):=\Gamma\left(V_{1}(\kappa)\right)$. The operator $V_{\mathrm{c}}(\kappa)$ obviously commutes with $T_{\mathrm{c}}$ and preserves $\Omega_{\mathrm{c}}$. Moreover, for field operators one has

$$
\begin{aligned}
& \operatorname{Ad} V_{\mathrm{c}}(\kappa)(\phi(f) \otimes \mathbb{1})=\cos 2 \pi \kappa(\phi(f) \otimes \mathbb{1})+\sin 2 \pi \kappa(\mathbb{1} \otimes \phi(f)), \\
& \operatorname{Ad} V_{\mathrm{c}}(\kappa)(\mathbb{1} \otimes \phi(g))=-\sin 2 \pi \kappa(\phi(g) \otimes \mathbb{1})+\cos 2 \pi \kappa(\mathbb{1} \otimes \phi(g)),
\end{aligned}
$$

and thus for exponentials it holds that $\operatorname{Ad} V_{\mathrm{c}}(\kappa)\left(e^{i(\phi(f) \otimes \mathbb{1})}\right)=e^{i \cos 2 \pi \kappa \phi(f)} \otimes$ $e^{i \sin 2 \pi \kappa \phi(f)}$ and $\operatorname{Ad} V_{\mathrm{c}}(\kappa)\left(e^{i(\mathbb{1} \otimes \phi(g))}\right)=e^{-i \sin 2 \pi \kappa \phi(g)} \otimes e^{i \cos 2 \pi \kappa \phi(g)}$. By considering $f$ and $g$ supported in $W_{\mathrm{R}}$, we conclude that $\operatorname{Ad} V_{\mathrm{c}}(\kappa)\left(\mathcal{M}_{\mathrm{c}}\right)=\mathcal{M}_{\mathrm{c}}$. In other words, $V_{\mathrm{c}}(\kappa)$ implements an inner symmetry of the group $\mathbb{R} / \mathbb{Z} \cong S^{1}$.

As we have seen in Section 4, our proof of strict locality works for an action of $S^{1}$. Hence in the following we consider only that case.

Since $\left(\mathcal{M}_{\mathrm{r}}, T_{\mathrm{r}}, \Omega_{\mathrm{r}}\right)$ is wedge-split, so is the tensor product $\left(\mathcal{M}_{\mathrm{c}}, T_{\mathrm{c}}, \Omega_{\mathrm{c}}\right)$. By considering the above action of $S^{1}$ by inner symmetry, one can construct Borchers triples $\left(\widetilde{\mathcal{M}}_{\mathrm{c}, \kappa}, \widetilde{T}_{\mathrm{c}}, \widetilde{\Omega}_{\mathrm{c}}\right)$ as in Section 3 , which we know to be strictly local by Section 4.

We first take a closer look at the action of $V_{\mathrm{c}}(\kappa)$. The matrix $V_{1}(\kappa)$ expressed above can be diagonalized by $\frac{1}{\sqrt{2}}\left(\begin{array}{cc}\mathbb{1} & i \mathbb{1} \\ \mathbb{1} & -i \mathbb{1}\end{array}\right)$ into $\left(\begin{array}{cc}e^{i 2 \pi \kappa} & 0 \\ 0 & e^{-i 2 \pi \kappa}\end{array}\right)$. Correspondingly we define $\mathcal{H}_{1, \pm}:=\left\{\psi \oplus \pm i \psi: \psi \in \mathcal{H}_{1}\right\}$. Then the full Fock space $\mathcal{H}_{\mathrm{c}}$ can be decomposed into $\mathbb{Z}$-graded subspaces $\mathcal{H}_{\mathrm{c}}=\bigoplus_{l \in \mathbb{Z}} \mathcal{H}_{\mathrm{c}}^{l}$ and we may assume that the generator $Q_{\mathrm{c}}$ of $V_{\mathrm{c}}(\kappa)$ acts by $l \mathbb{1}$ on $\mathcal{H}_{\mathrm{c}}^{l}$ (by definition of the grading), and $V_{\mathrm{c}}(\kappa)=e^{i 2 \pi \kappa Q_{\mathrm{c}}}$. Hence on the Hilbert space of interest to us, $\widetilde{\mathcal{H}}_{\mathrm{c}}=\mathcal{H}_{\mathrm{c}} \otimes \mathcal{H}_{\mathrm{c}}=\bigoplus \mathcal{H}_{\mathrm{c}}^{l} \otimes \mathcal{H}_{\mathrm{c}}^{m}$, it is clear that $Q_{\mathrm{c}} \otimes Q_{\mathrm{c}}$ acts by $\operatorname{lm} \mathbb{1}$ on $\mathcal{H}_{\mathrm{c}}^{l} \otimes \mathcal{H}_{\mathrm{c}}^{m}$.

Now the operator $b_{+}^{\dagger}(\psi):=b^{\dagger}(\psi \oplus(i \psi))$ on $\mathcal{H}_{\mathrm{c}}$ increments the grading and so does $b_{-}(\psi):=b(\psi \oplus(-i \psi))$. On the other hand, $b_{-}^{\dagger}(\psi):=b^{\dagger}(\psi \oplus(-i \psi))$ and $b_{+}(\psi):=b(\psi \oplus(i \psi))$ decrement the grading.

Now it is easy to see the following twisted commutation relations:

$$
\begin{aligned}
& b_{ \pm}^{\dagger}\left(\psi_{1}\right) \otimes \mathbb{1} \cdot \operatorname{Ad} \widetilde{V}_{\mathrm{c}, \kappa}\left(\mathbb{1} \otimes b_{ \pm}^{\dagger}\left(\psi_{2}\right)\right) \\
& \quad-e^{ \pm(\mp i 2 \pi \kappa)} \operatorname{Ad} \widetilde{V}_{\mathrm{c}, \kappa}\left(\mathbb{1} \otimes b_{ \pm}^{\dagger}\left(\psi_{2}\right)\right) \cdot b_{ \pm}^{\dagger}\left(\psi_{1}\right) \otimes \mathbb{1}=0 \\
& b_{ \pm}\left(\psi_{1}\right) \otimes \mathbb{1} \cdot \operatorname{Ad} \widetilde{V}_{\mathrm{c}, \kappa}\left(\mathbb{1} \otimes b_{ \pm}^{\dagger}\left(\psi_{2}\right)\right) \\
& \quad-e^{\mp(\mp i 2 \pi \kappa)} \operatorname{Ad} \widetilde{V}_{\mathrm{c}, \kappa}\left(\mathbb{1} \otimes b_{ \pm}^{\dagger}\left(\psi_{2}\right)\right) \cdot b_{ \pm}\left(\psi_{1}\right) \otimes \mathbb{1}=0 \\
& b_{ \pm}^{\dagger}\left(\psi_{1}\right) \otimes \mathbb{1} \cdot \operatorname{Ad} \widetilde{V}_{\mathrm{c}, \kappa}\left(\mathbb{1} \otimes b_{ \pm}\left(\psi_{2}\right)\right) \\
& \quad-e^{ \pm( \pm i 2 \pi \kappa)} \operatorname{Ad} \widetilde{V}_{\mathrm{c}, \kappa}\left(\mathbb{1} \otimes b_{ \pm}\left(\psi_{2}\right)\right) \cdot b_{ \pm}^{\dagger}\left(\psi_{1}\right) \otimes \mathbb{1}=0
\end{aligned}
$$




$$
\begin{aligned}
& b_{ \pm}\left(\psi_{1}\right) \otimes \mathbb{1} \cdot \operatorname{Ad} \widetilde{V}_{\mathrm{c}, \kappa}\left(\mathbb{1} \otimes b_{ \pm}\left(\psi_{2}\right)\right) \\
& \quad-e^{\mp( \pm i 2 \pi \kappa)} \operatorname{Ad} \widetilde{V}_{\mathrm{c}, \kappa}\left(\mathbb{1} \otimes b_{ \pm}\left(\psi_{2}\right)\right) \cdot b_{ \pm}\left(\psi_{1}\right) \otimes \mathbb{1}=0
\end{aligned}
$$

where the signs \pm and so on in the first term correspond to respectively to \pm and so on in the constant factor in the second term. The commutation relation between objects with or without Ad $\widetilde{V}_{\mathrm{c}, \kappa}$ follows trivially from the usual ones. Namely, we have

$$
\left[b_{ \pm}^{\dagger}\left(\psi_{1}\right), b_{ \pm}^{\dagger}\left(\psi_{2}\right)\right]=0, \quad\left[b_{ \pm}^{\dagger}\left(\psi_{1}\right), b_{ \pm}\left(\psi_{2}\right)\right]=\left\langle\psi_{2}, \psi_{1}\right\rangle \mathbb{1}
$$

and all other combinations commute (note that $\langle\cdot, \cdot\rangle$ is linear in the second argument and $b^{\dagger}(\cdot)$ is linear and $b(\cdot)$ is antilinear). In other words, these operatorvalued distributions satisfy the Zamolodchikov-Fadeev algebra, with the $S$-matrix given by the phase factors. This two-particle scattering matrix (see below) does not depend on the rapidity $\theta=\log p$ (note that $p$ is associated with the lightlike translation, not the spacelike translation as usual. We will assume that the mass is 1 for simplicity).

Note that this set of commutation relations can be summarized in the form of matrix. We take a basis $\left\{e_{1,+}, e_{1,-}, e_{2,+}, e_{2,-}\right\}$ on $\mathbb{C}^{2} \otimes \mathbb{C}^{2}$ and accordingly $\left\{e_{1,+} \otimes\right.$ $\left.e_{1,+}, e_{1,+} \otimes e_{1,-}, e_{1,+} \otimes e_{2,+}, e_{1,+} \otimes e_{2,-}, \ldots\right\}$ on $\left(\mathbb{C}^{2} \otimes \mathbb{C}^{2}\right) \otimes\left(\mathbb{C}^{2} \otimes \mathbb{C}^{2}\right)$, where

$$
\left(\mathcal{H}_{\mathrm{r}} \oplus \mathcal{H}_{\mathrm{r}}\right) \oplus\left(\mathcal{H}_{\mathrm{r}} \oplus \mathcal{H}_{\mathrm{r}}\right)=\left(\mathcal{H}_{\mathrm{r}} \otimes \mathbb{C}^{2}\right) \otimes \mathbb{C}^{2}
$$

is understood. The signs \pm refer to the structure of the complex free field, while indices 1,2 are the first and the second copies of the field. The two-particle $S$-matrix $\widetilde{S}_{\mathrm{c}, \kappa}(\theta)$ is given on this basis by (note that this is constant with respect to $\theta$ )

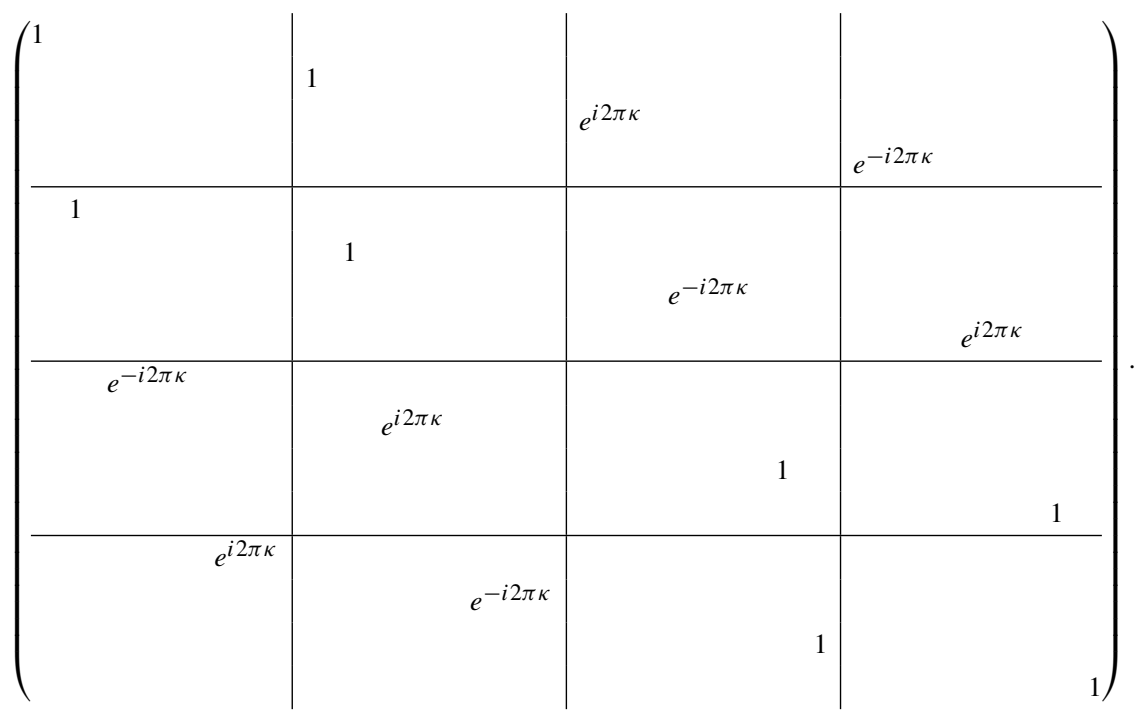


And it is straightforward to see that this complies the conditions of [25, Definition 2.1] if the charge conjugation $J_{\mathrm{c}}$ is introduced which exchanges + and - fields in each component. This is said to be diagonal in the sense of [25, Section 6]. The advantage of our methods is that the strict locality can be seen as an immediate consequence of Section 4.

This two-particle $S$-matrix is nontrivial only for between different components. One could say that the interaction occurs only between particles of different species but there is no self-interaction. This is clear also from the construction: one component remains unchanged and the other component is just shifted by a unitary equivalence; thus the twisting exists only between different components.

With the help of the analysis [25], we have the following, since we see below that our von Neumann algebra is generated by those wedge-local fields.

THEOREM 5.1. The triple $\left(\widetilde{\mathcal{M}}_{\mathrm{c}, \kappa}, \widetilde{T}_{\mathrm{c}}, \widetilde{\Omega}_{\mathrm{c}}\right)$ is strictly local and the corresponding Haag-Kastler net is asymptotically complete and interacting, and the S-matrix is factorizing and its two-particle S-matrix is given as above.

Comparison of von Neumann algebras. By definition we have $\widetilde{\mathcal{M}}_{\mathrm{c}, \kappa}=\mathcal{M}_{\mathrm{c}} \otimes \mathbb{1} \vee$ Ad $\widetilde{V}_{\mathrm{c}, \kappa}\left(\mathbb{1} \otimes \mathcal{M}_{\mathrm{c}}\right)$ and $\mathcal{M}_{\mathrm{c}}$ is generated by the exponential of fields $\phi(f) \otimes \mathbb{1}, \mathbb{1} \otimes$ $\phi(g)$, where $f, g$ are real test functions with supp $f$, supp $g \subset W_{\mathrm{R}}$ and $\mathcal{H}_{\mathrm{c}}=\mathcal{H}_{\mathrm{r}} \otimes$ $\mathcal{H}_{\mathrm{r}}$ is understood. First let us consider the $\mathcal{M}_{\mathrm{c}} \otimes \mathbb{1}$ component. Following [25], we consider pairs of complex-valued test functions $f, g$ such that $\bar{f}=g$. The complex field which generates the wedge algebra in [25] is given in our notation by

$$
\begin{aligned}
\phi_{\mathrm{c}}(f \oplus g) & =b_{\mathrm{c}}^{\dagger}\left(f^{+} \oplus g^{+}\right)+b_{\mathrm{c}}\left(J_{1}\left(f^{-} \oplus g^{-}\right)\right) \\
& :=b_{+}^{\dagger}\left(f^{+}\right)+b_{-}^{\dagger}\left(g^{+}\right)+b_{+}\left(\overline{g^{-}}\right)+b_{-}\left(\overline{f^{-}}\right) \\
& =\phi(f) \otimes \mathbb{1}+\mathbb{1} \otimes i \phi(f)+\phi(g) \otimes \mathbb{1}-\mathbb{1} \otimes i \phi(g) \\
& =\phi(f+\bar{f}) \otimes \mathbb{1}+\mathbb{1} \otimes i \phi(f-\bar{f}) \\
& =\phi(2 \operatorname{Re} f) \otimes \mathbb{1}-\mathbb{1} \otimes \phi(2 \operatorname{Im} f),
\end{aligned}
$$

where $J_{1}(\xi \oplus \eta)=\bar{\eta} \oplus \bar{\xi}$ and $\xi, \eta \in L^{2}(\mathbb{R}, d \theta)$ and $f^{ \pm}(\theta), g^{ \pm}(\theta)$ are defined as before. Namely, the fields in complex and real bases are just the linear combinations of each other. Note that $\phi$ and $b^{\dagger}$ are linear but $b$ is antilinear.

It follows also that $\operatorname{Ad} \widetilde{V}_{\mathrm{c}, \kappa}\left(\mathbb{1} \otimes \phi_{\mathrm{c}}(f \oplus g)\right)=\operatorname{Ad} \widetilde{V}_{\mathrm{c}, \kappa}\left(\mathbb{1} \otimes\left(\phi_{\mathrm{c}}(2 \operatorname{Re} f) \otimes \mathbb{1}-\right.\right.$ $\left.\left.\mathbb{1} \otimes \phi_{\mathrm{c}}(2 \operatorname{Im} f)\right)\right)$. From this one easily shows that the wedge algebra generated by the Zamolodchikov-Fadeev fields in the sense of [25] is equal to $\widetilde{\mathcal{M}}_{\mathrm{c}, \kappa}=$ $\mathcal{M}_{\mathrm{c}} \otimes \mathbb{1} \vee \operatorname{Ad} \widetilde{V}_{\mathrm{c}, \kappa}\left(\mathbb{1} \otimes \mathcal{M}_{\mathrm{c}}\right)$.

The relation to the Federbush model. One notices that this $S$-matrix is very similar to the one of the Federbush model $[14,29,31]$, although here the fields 
are bosonic. However,our procedure can be easily adapted to fermionic nets. Moreover, in the traditional approach there were technical problems: one can construct local fields only for small coupling constant [30], or if one takes the bootstrap approach, the convergence of form factors is not clear [1]. Here this problem is completely solved. We can prove the existence of local operators for any value of $\kappa$ if we consider the action of $S^{1}$. By comparing the $S$-matrix, this corresponds to an arbitrary value of the coupling constant.

More importantly, our construction is not restricted to the Federbush models. One can take any wedge-split net with inner symmetry. This contains, for example, the tensor product of one of Lechner's models [22], instead of the real free field. One can consider $n$ copies of the real free field, which have $\mathrm{O}(n)$ symmetry, then take any subgroup of $\mathrm{O}(n)$ isomorphic to $S^{1}$ or $\mathbb{Z}_{N}$. This should correspond to the Lie-algebraic generalization of the Federbush models, whose form factors were proposed in [14]. It works also with $n$ copies of one of Lechner's models. Furthermore, the constructed net admits again inner symmetry and is wedge-split; hence one can repeat the construction to obtain further new models (on a bigger Hilbert space).

Some technical remarks on inner symmetry. The wedge algebra of our twodimensional nets is given by the tensor product twisted by the inner symmetry. However, this does not mean that there is a subnet which is a copy of a tensor component. This is clear because any tensor component in the right wedge does not commute with the left wedge unless it is in the fixed point with respect to the action of $S^{1}$. It is also noted that a wedge-split net has no nontrivial DHR sector [28]; hence any extension of such a net is a tensor product.

One realizes that the whole net still admits an action of $S^{1} \times S^{1}$ by inner symmetry. The fixed point net may fail to have Haag duality [28] and the standard sector theory does not apply. The whole net is an extension of this fixed point net.

Our models violate the parity symmetry, which is clear from the $S$-matrix. However, the extended parity which interchanges the two components is preserved (see [31, Section 6.3.3]). As noted in [10, Theorem 3.3], the parity symmetry is essential for the Bisognano-Wichmann property in two dimensions. Although this is not necessarily related to our models, we present a simple counterexample. One takes the complex free field, which admits $S^{1}$-inner symmetry with the Bisognano-Wichmann property. Then one can simply replace the Lorentz boosts by the composition of Lorentz boosts and the inner symmetry. This still satisfies all the axioms of the net but violates the parity symmetry which must have the appropriate commutation relation with the boosts. Accordingly, the inner symmetry and Poincaré symmetry do not necessarily commute. For example, we can take a net with a noncommutative Lie group symmetry and replace the boosts as above. The proof in four dimensions [15, Theorem 10.4] 
does not work in two dimensions since the Lorentz group is abelian, and hence the Poincaré group has finite dimensional unitary representations.

\section{Borchers triples through Longo-Witten endomorphisms on the U(1)-current net}

Here we exhibit another procedure for producing Borchers triples in a more concrete way. We take the free massive net as the starting point (Section 2.3). The formulas are quite similar to those in [34], but should not be confused. Strict locality of the models constructed here is not investigated in the present paper. The author expects that the wedge-local field presentation in Section 6.3 would help in proving strict locality for the construction here.

6.1. Reduction to a light ray. Let $\left(\mathcal{A}_{\mathrm{r}}, U_{\mathrm{r}}, \Omega_{\mathrm{r}}\right)$ be the free massive net. The representation $U_{\mathrm{r}}$ can be restricted to the positive light ray, which we denote by $U_{\mathrm{r}}^{+}$, and we obtain a one-dimensional Borchers triple $\left(\mathcal{M}_{\mathrm{r}}, U_{\mathrm{r}}^{+}, \Omega_{\mathrm{r}}\right)$, namely a von Neumann algebra $\mathcal{M}_{\mathrm{r}}:=\mathcal{A}_{\mathrm{r}}\left(W_{\mathrm{R}}\right)$, a positive energy representation $U_{\mathrm{r}}^{+}$ of $\mathbb{R}$ and a cyclic separating vector $\Omega_{\mathrm{r}}$ for $\mathcal{A}_{\mathrm{r}}$ invariant under $U_{\mathrm{r}}^{+}$such that Ad $U_{\mathrm{r}}^{+}(t)\left(\mathcal{M}_{\mathrm{r}}\right) \subset \mathcal{M}_{\mathrm{r}}$ for $t \in \mathbb{R}_{+}$. We denote the translation along the negative light ray by $U_{\mathrm{r}}^{-}$.

Let us recall the $\mathrm{U}(1)$-current net $\left(\mathcal{A}^{(0)}, U_{0}, \Omega_{0}\right)$, which is a conformal net. For its definition, see our previous discussion [34, Section 5]. The point is that the Hilbert space is naturally isomorphic to the Fock space $\mathcal{H}_{\mathrm{r}}$ of the massive free net, whose one-particle space is $L^{2}(\mathbb{R}, d \theta)$, and one considers the second-quantization operators. The above one-dimensional triple $\left(\mathcal{M}_{\mathrm{r}}, U_{\mathrm{r}}^{+}, \Omega_{\mathrm{r}}\right)$ is actually unitarily equivalent to the triple $\left(\mathcal{A}^{(0)}\left(\mathbb{R}_{+}\right), T_{0}, \Omega_{0}\right)$, where $\left(\mathcal{A}^{(0)}, U_{0}, \Omega_{0}\right)$ is the $\mathrm{U}(1)$ current net and $T_{0}$ is the restriction of $U_{0}$ to the translation subgroup. This will be explained in more detail in [5].

In particular, we can exploit the Longo-Witten endomorphisms found in [27]. Recall that, for an inner symmetric function $\varphi(z)$, namely the boundary value on $\mathbb{R}$ of a bounded analytic function on $0<\operatorname{Im} z<\pi$, one considers the operator $V_{\varphi}:=\Gamma\left(\varphi\left(P_{1}\right)\right)$, where $P_{1}$ is the generator of the restriction of $U_{\mathrm{r}}^{+}$on the oneparticle space $\mathcal{H}_{1}, \varphi\left(P_{1}\right)$ denotes the operator defined by functional calculus and $\Gamma$ is the second quantization (note that in general the order of second quantization and functional calculus cannot be exchanged: $\left.\Gamma\left(\varphi\left(P_{1}\right)\right) \neq \varphi\left(\Gamma\left(P_{1}\right)\right)\right)$.

Then $\operatorname{Ad} V_{\varphi}$ preserves $\mathcal{M}_{\mathrm{r}}$ and $V_{\varphi}$ commutes with $U_{\mathrm{r}}^{+}$. Furthermore, $V_{\varphi}$ commutes with $U_{\mathrm{r}}^{-}$since $U_{\mathrm{r}}^{-}(a)=\Gamma\left(\exp \left(\frac{i t}{P_{1}}\right)\right)$, as we see in [5].

6.2. Construction of Borchers triples. We work on the tensor product Hilbert space $\widetilde{\mathcal{H}}_{\mathrm{r}}:=\mathcal{H}_{\mathrm{r}} \otimes \mathcal{H}_{\mathrm{r}}$. We fix an inner symmetric function $\varphi$. As above, $P_{1}$ is the one-particle lightlike translation. Let us recall our argument [34, Section 5]. 
The physical Hilbert space $\mathcal{H}_{\mathrm{r}}$ is included in the unsymmetrized Fock space $\mathcal{H}^{\Sigma}$. We consider $m$ commuting operators on $\mathcal{H}_{1}^{\otimes m}$ :

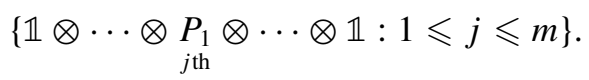

For $1 \leqslant j \leqslant m$ and $1 \leqslant k \leqslant n$, let us define operators on $\mathcal{H}^{m} \otimes \mathcal{H}^{n}$ :

$$
\begin{aligned}
P_{j, k}^{m, n} & :=\left(\mathbb{1} \otimes \cdots \otimes \frac{1}{P_{1}} \otimes \cdots \otimes \mathbb{1}\right) \otimes\left(\mathbb{1} \otimes \cdots \otimes P_{k \text { th }} \otimes \cdots \otimes \mathbb{1}\right) \\
R_{\varphi}^{m, n} & :=\prod_{j, k} \varphi\left(P_{j, k}^{m, n}\right),
\end{aligned}
$$

where $\varphi\left(P_{j, k}^{m, n}\right)$ is defined by functional calculus on $\mathcal{H}_{1}^{\otimes m} \otimes \mathcal{H}_{1}^{\otimes n}$. Now, our key operator on the unsymmetrized space $\mathcal{H}^{\Sigma} \otimes \mathcal{H}^{\Sigma}$ is

$$
\widetilde{R}_{\varphi}:=\bigoplus_{m, n} R_{\varphi}^{m, n}=\bigoplus_{m, n} \prod_{j, k} \varphi\left(P_{j, k}^{m, n}\right),
$$

where for $m=0$ or $n=0$ we set $R_{j, k}^{m, n}=\mathbb{1}$ as a convention. It is easy to see that $\widetilde{R}_{\varphi}$ naturally restricts to partially symmetrized subspaces $\mathcal{H}_{\mathrm{r}} \otimes \mathcal{H}^{\Sigma}$ and $\mathcal{H}^{\Sigma} \otimes \mathcal{H}_{\mathrm{r}}$ and to the totally symmetrized space $\mathcal{H}_{\mathrm{r}} \otimes \mathcal{H}_{\mathrm{r}}$.

Let $E_{1} \otimes E_{1} \otimes \cdots \otimes E_{1}$ be the joint spectral measure of $\left\{\mathbb{1} \otimes \cdots \otimes P_{1} \otimes \cdots \otimes \mathbb{1}\right.$ : $1 \leqslant k \leqslant n\}$. One obtains the following expression:

$$
\begin{aligned}
\varphi\left(P_{j, k}^{m, n}\right) & =\int\left(\mathbb{1} \otimes \cdots \otimes \varphi(\underbrace{p_{k}}_{j \mathrm{th}} P_{1}) \otimes \cdots \mathbb{1}\right) \otimes\left(\mathbb{1} \otimes \cdots d E_{k \mathrm{th}}\left(p_{k}\right) \otimes \cdots \mathbb{1}\right) \\
& =\int\left(\underset{j \mathrm{th}}{\mathbb{1} \otimes \cdots d E_{1}\left(p_{j}\right) \otimes \cdots \mathbb{1}}\right) \otimes\left(\mathbb{1} \otimes \cdots \otimes \varphi\left(\frac{P_{1}}{p_{j}}\right) \otimes \cdots \mathbb{1}\right) .
\end{aligned}
$$

Like for our previous case [34, Section 5.2], we decompose $\widetilde{R}_{\varphi}$ with respect only to the right or left component:

$$
\begin{aligned}
\widetilde{R}_{\varphi} & =\bigoplus_{n} \int \prod_{k} \Gamma\left(\varphi\left(\frac{p_{k}}{P_{1}}\right)\right) \otimes d E_{1}\left(p_{1}\right) \otimes \cdots \otimes d E_{1}\left(p_{n}\right) \\
& =\bigoplus_{m} \int d E_{1}\left(p_{1}\right) \otimes \cdots \otimes d E_{1}\left(p_{m}\right) \otimes \prod_{j} \Gamma\left(\varphi\left(\frac{P_{1}}{p_{j}}\right)\right) .
\end{aligned}
$$


For the proof, we refer the reader to [34, Section 5.2]. The expression in the first line naturally restricts to the partially symmetrized space $\mathcal{H}_{\mathrm{r}} \otimes \mathcal{H}^{\Sigma}$ and the second expression to $\mathcal{H}^{\Sigma} \otimes \mathcal{H}_{\mathrm{r}}$.

We have a variant of [34, Lemma 5.2].

LEMMA 6.1. It holds for $x \in \mathcal{A}_{\mathrm{r}}\left(W_{\mathrm{R}}\right)$ and $x^{\prime} \in \mathcal{A}_{\mathrm{r}}\left(W_{\mathrm{R}}\right)^{\prime}$ that

$$
\left[x \otimes \mathbb{1}, \operatorname{Ad} \widetilde{R}_{\varphi}\left(x^{\prime} \otimes \mathbb{1}\right)\right]=0
$$

on the Hilbert space $\mathcal{H}_{\mathrm{r}} \otimes \mathcal{H}_{\mathrm{r}}$. Similarly, for $y \in \mathcal{A}_{\mathrm{r}}\left(W_{\mathrm{R}}\right)$ and $y^{\prime} \in \mathcal{A}_{\mathrm{r}}\left(W_{\mathrm{R}}\right)^{\prime}$ one has

$$
\left[\text { Ad } \widetilde{R}_{\varphi}(\mathbb{1} \otimes y), \mathbb{1} \otimes y^{\prime}\right]=0 .
$$

Proof. The operator $\widetilde{R}_{\varphi}$ is disintegrated into second-quantization operators as we saw above. First we consider the first of the commutators above. The operator $\widetilde{R}_{\varphi}$ restricts naturally to $\mathcal{H}_{\mathrm{r}} \otimes \mathcal{H}^{\Sigma}$, and $x \otimes \mathbb{1}$ and $x^{\prime} \otimes \mathbb{1}$ extend naturally to $\mathcal{H}_{\mathrm{r}} \otimes \mathcal{H}^{\Sigma}$. It is easy to see that if $\varphi(z)$ is an inner symmetric function, then so are $\overline{\varphi(1 / \bar{z})}$ and hence $\overline{\varphi\left(p_{k} / \bar{z}\right)}$ for $p_{k} \geqslant 0$. The first commutation relation is equivalent to

$$
\left[\text { Ad } \widetilde{R}_{\bar{\varphi}}(x \otimes \mathbb{1}), x^{\prime} \otimes \mathbb{1}\right]=0 .
$$

Let us prove this on $\mathcal{H}_{\mathrm{r}} \otimes \mathcal{H}^{\Sigma}$. We have

$\operatorname{Ad} \widetilde{R}_{\bar{\varphi}}(x \otimes \mathbb{1})=\bigoplus_{n} \int \operatorname{Ad}\left(\prod_{k} \Gamma\left(\bar{\varphi}\left(\frac{p_{k}}{P_{1}}\right)\right)\right)(x) \otimes d E_{1}\left(p_{1}\right) \otimes \cdots \otimes d E_{1}\left(p_{n}\right)$, and this commutes with $x^{\prime} \otimes \mathbb{1}$. Indeed, since $x \in \mathcal{A}_{\mathrm{r}}\left(W_{\mathrm{R}}\right)=\mathcal{A}_{0}\left(\mathbb{R}_{+}\right)$and $x^{\prime} \in$ $\mathcal{A}_{\mathrm{r}}\left(W_{\mathrm{R}}\right)^{\prime}=\mathcal{A}_{0}\left(\mathbb{R}_{-}\right)$, it follows that $\operatorname{Ad} \Gamma\left(\bar{\varphi}\left(\frac{p_{k}}{P_{1}}\right)\right)(x) \in \mathcal{A}_{0}\left(\mathbb{R}_{+}\right)$for any $p_{k} \geqslant 0$ by the result of Longo and Witten [27] (see also the beginning of this section), and by the fact that the spectral support of $E_{1}$ is positive. Now the commutation relation just proved naturally restricts to $\mathcal{H}_{\mathrm{r}} \otimes \mathcal{H}_{\mathrm{r}}$ and we obtain the first relation.

The proof of the second commutation relation goes more simply. We only have to consider the expression

$\operatorname{Ad} \widetilde{R}_{\varphi}(\mathbb{1} \otimes y)=\bigoplus_{m} \int d E_{1}\left(p_{1}\right) \otimes \cdots \otimes d E_{1}\left(p_{m}\right) \otimes \operatorname{Ad}\left(\prod_{j} \Gamma\left(\varphi\left(\frac{P_{1}}{p_{j}}\right)\right)\right)(y)$.

The rest of the argument is parallel to that above.

Let $T_{\mathrm{r}}$ be the restriction of $U_{\mathrm{r}}$ to translation (not to be confused with trace). Our Borchers triple is given as follows. 
THEOREM 6.2. The triple
(1) $\widetilde{\mathcal{M}}_{\mathrm{r}, \varphi}:=\left\{x \otimes \mathbb{1}, \text { Ad } \widetilde{R}_{\varphi}(\mathbb{1} \otimes y): x, y \in \mathcal{A}_{\mathrm{r}}\left(W_{\mathrm{R}}\right)\right\}^{\prime \prime}$
(2) $\widetilde{T}_{\mathrm{r}}=T_{\mathrm{r}} \otimes T_{\mathrm{r}}$
(3) $\widetilde{\Omega}_{\mathrm{r}}=\Omega_{\mathrm{r}} \otimes \Omega_{\mathrm{r}}$

is a Borchers triple.

Proof. The conditions on $\widetilde{T}_{\mathrm{r}}$ and $\widetilde{\Omega}_{\mathrm{r}}$ are readily satisfied since they are same as the tensor product net. The operators $\widetilde{R}_{\varphi}$ and $\widetilde{T}_{\mathrm{r}}$ commute since both are defined by the functional calculus of the same spectral measure (recall that $T_{\mathrm{r}}\left(t_{+}, t_{-}\right)=$ $\left.U_{\mathrm{r}}^{+}\left(t_{+}\right) U_{\mathrm{r}}^{-}\left(t_{-}\right)\right)$; hence $\widetilde{T}_{\mathrm{r}}\left(t_{+}, t_{-}\right)$sends $\widetilde{\mathcal{M}}_{\mathrm{r}, \varphi}$ into itself for $\left(t_{+}, t_{-}\right) \in W_{\mathrm{R}}$. The vector $\widetilde{\Omega}_{\mathrm{r}}$ is cyclic for $\widetilde{\mathcal{M}}_{\mathrm{r}, \varphi}$ since $\widetilde{\mathcal{M}}_{\mathrm{r}, \varphi} \widetilde{\Omega}_{\mathrm{r}} \supset\left\{(x \otimes \mathbb{1}) \cdot \widetilde{R}_{\varphi} \cdot(\mathbb{1} \otimes y) \cdot \widetilde{\Omega}_{\mathrm{r}}\right\}=$ $\left\{(x \otimes \mathbb{1}) \cdot(\mathbb{1} \otimes y) \cdot \widetilde{\Omega}_{\mathrm{r}}\right\}$ and the latter is total by the Reeh-Schlieder property of the tensor product net.

We see the separating property of $\widetilde{\Omega}_{\mathrm{r}}$ as follows. Consider a von Neumann algebra

$$
\widetilde{\mathcal{M}}_{\mathrm{r}, \varphi}^{1}:=\left\{\operatorname{Ad} \widetilde{R}_{\varphi}\left(x^{\prime} \otimes \mathbb{1}\right), \mathbb{1} \otimes y^{\prime}: x^{\prime}, y^{\prime} \in \mathcal{A}_{\mathrm{r}}\left(W_{\mathrm{R}}\right)^{\prime}\right\}^{\prime \prime}
$$

One verifies that $\widetilde{\Omega}_{\mathrm{r}}$ is cyclic for $\widetilde{\mathcal{M}}_{\mathrm{r}, \varphi}^{1}$ as above; hence we only have to show that $\widetilde{\mathcal{M}}_{\mathrm{r}, \varphi}$ and $\widetilde{\mathcal{M}}_{\mathrm{r}, \varphi}^{1}$ commute. This has been done by Lemma 6.1 .

One can actually show that $\widetilde{\mathcal{M}}_{\mathrm{r}, \varphi}^{1}=\left(\widetilde{\mathcal{M}}_{\mathrm{r}, \varphi}\right)^{\prime}$, so this confusing notation is justified. Indeed, one has only to check that the modular group of $\widetilde{\mathcal{M}}_{\mathrm{r}, \varphi}$ with respect to $\widetilde{\Omega}_{\mathrm{r}}$ is the same for the tensor product, which follows from the field representation in the next section and the argument of [23].

In this presentation we took $\mathcal{A}_{\mathrm{r}}$ as the starting point. It is also possible to take the models in [22] or more general models with spectra with more particles [25]. We will discuss this (slight) generalization in [5]. In this paper we do not consider strict locality of these Borchers triples, although the author expects that a proof similar to that of [22] should work.

6.3. Realization as deformed fields. Let us see that the Borchers triples constructed in Section 6 admit a wedge-local field interpretation. Since the twisting operator $\widetilde{R}_{\varphi}$ is given on each particle number space, the calculation is straightforward. We only need the following commutation relations:

$$
\begin{aligned}
b^{\dagger}\left(\psi_{1}\right) \otimes & \mathbb{1} \cdot \operatorname{Ad} \widetilde{R}_{\varphi}\left(\mathbb{1} \otimes b^{\dagger}\left(\psi_{2}\right)\right) \\
& -\int d p d p^{\prime} \psi_{1}(p) \psi_{2}\left(p^{\prime}\right) \varphi\left(\frac{p}{p^{\prime}}\right) \operatorname{Ad} \widetilde{R}_{\varphi}\left(\mathbb{1} \otimes b^{\dagger}\left(p^{\prime}\right)\right) \cdot b^{\dagger}(p) \otimes \mathbb{1}=0,
\end{aligned}
$$


or, with rapidity $\theta=\log p$ (here again $p$ is associated with the lightlike translation as above and the mass is 1) and in terms of operator-valued distributions, one has

$$
b^{\dagger}(\theta) \otimes \mathbb{1} \cdot \operatorname{Ad} \widetilde{R}_{\varphi}\left(\mathbb{1} \otimes b^{\dagger}\left(\theta^{\prime}\right)\right)-\varphi\left(e^{\theta-\theta^{\prime}}\right) \operatorname{Ad} \widetilde{R}_{\varphi}\left(\mathbb{1} \otimes b^{\dagger}\left(\theta^{\prime}\right)\right) \cdot b^{\dagger}(\theta) \otimes \mathbb{1}=0 .
$$

Note that if $\varphi$ is an inner symmetric function, then $\varphi\left(e^{\theta}\right)$ is a bounded analytic function in the strip $0<\operatorname{Im} \theta<\pi$ and $\varphi\left(e^{i \pi-\theta}\right)=\varphi\left(-e^{-\theta}\right)$ has the same property. In the matrix form, this can be written as

$$
\widetilde{S}_{\mathrm{r}, \varphi}(\theta)=\left(\begin{array}{llll}
1 & & & \\
& & \varphi\left(e^{\theta}\right) & \\
& \varphi\left(-e^{-\theta}\right) & & \\
& & & 1
\end{array}\right)
$$

on the basis $\left\{e_{1} \otimes e_{1}, e_{1} \otimes e_{2}, e_{2} \otimes e_{1}, e_{2} \otimes e_{2}\right\}$. This is again a diagonal $S$-matrix. From its simple form, it is expected that the proof of modular nuclearity is similar to the one in [22].

It is clear that also in these models, the interaction occurs only between different components.

\section{Summary and outlook}

In this paper, we presented a novel procedure for obtaining interacting quantum field models realized as nets of observables. One can view this procedure as first preparing a pair of models and then making them couple.

The method is thoroughly operator-algebraic and in the most abstract setting of Section 4 no field picture (wedge-local or not) is required. On the other hand, the interaction is fairly weak. When the field description is available, a pair of particles of different species obtains a phase during the interaction and no momentum transfer occurs. A connection with the wedge-local field approach and our previously constructed Borchers triples has been found in [24]. It is worth investigating how to obtain more general integrable models (see for example [22, 25]) purely operator-algebraically, where a 'self-interaction' occurs.

Furthermore, an interesting variant has been obtained in [6]. Strict locality of the models therein is not known, but the $S$-matrix shows a phenomenon which resembles particle production. It is desired to establish strict locality of these models or to find massive counterparts.

More ambitiously, certain relations are claimed between integrable models and gauge theories of higher dimension (see for example [4]). The author wishes to study such connections with an analytical approach. 


\section{Acknowledgements}

I would like to thank Marcel Bischoff and Karl-Henning Rehren for helpful discussions.

I gratefully acknowledge the support by the Deutscher Akademischer Austauschdienst, Hausdorff Institut für Mathematik, Alexander von Humboldt Stiftung and Grant-in-Aid for JSPS fellows 25-205, and partial support by the Courant Research Center 'Higher Order Structures in Mathematics', Göttingen.

\section{References}

[1] H. Babujian and M. Karowski, 'Towards the construction of Wightman functions of integrable quantum field theories', in Proceedings of 6th International Workshop on Conformal Field Theory and Integrable Models, 19, 2004, 34-49.

[2] V. Bargmann, 'On unitary ray representations of continuous groups', Ann. of Math. (2) 59 (1954), 1-46.

[3] H. Baumgärtel, Operator Algebraic Methods in Quantum Field Theory, (Akademie Verlag, Berlin, 1995).

[4] N. Beisert et al., 'Review of AdS/CFT integrability: an overview', Lett. Math. Phys. 99 (2012), 3-32.

[5] M. Bischoff and Y. Tanimoto, 'Integrable QFT and Longo-Witten endomorphisms', Ann. Henri Poincare (2014) (to appear).

[6] M. Bischoff and Y. Tanimoto, 'Construction of wedge-local nets of observables through Longo-Witten endomorphisms. II’, Commun. Math. Phys. 317 (2013), 667-695.

[7] H.-J. Borchers, 'The CPT-theorem in two-dimensional theories of local observables', Commun. Math. Phys. 143 (1992), 315-332.

[8] H. -J. Borchers, D. Buchholz and B. Schroer, 'Polarization-free generators and the $S$-matrix', Commun. Math. Phys. 219 (2001), 125-140.

[9] O. Bratteli and D. W. Robinson, Operator Algebras and Quantum Statistical Mechanics. 2. Equilibrium States. Models in Quantum Statistical Mechanics, 2nd edn, Texts and Monographs in Physics (Springer-Verlag, Berlin, 1997).

[10] R. Brunetti, D. Guido and R. Longo, 'Modular structure and duality in conformal quantum field theory', Commun. Math. Phys. 156 (1993), 201-219.

[11] D. Buchholz, C. D'Antoni and R. Longo, 'Nuclear maps and modular structures. I. General properties', J. Funct. Anal. 88 (1990), 233-250.

[12] D. Buchholz and G. Lechner, 'Modular nuclearity and localization', Ann. Henri Poincaré 5 (2004), 1065-1080.

[13] D. Buchholz, G. Lechner and S. J. Summers, 'Warped convolutions, Rieffel deformations and the construction of quantum field theories', Commun. Math. Phys. 304 (2011), 95-123.

[14] O. A. Castro-Alvaredo and A. Fring, 'Form factors from free fermionic Fock fields, the Federbush model', Nucl. Phys. B 618 (2001), 437-464.

[15] S. Doplicher and R. Longo, 'Standard and split inclusions of von Neumann algebras', Invent. Math. 75 (1984), 493-536.

[16] S. Doplicher and J. E. Roberts, 'A new duality theory for compact groups', Invent. Math. 98 (1989), 157-218. 
[17] S. Doplicher and J. E. Roberts, 'Why there is a field algebra with a compact gauge group describing the superselection structure in particle physics', Commun. Math. Phys. 131 (1990), 51-107.

[18] W. Dybalski and Y. Tanimoto, 'Asymptotic completeness in a class of massless relativistic quantum field theories', Commun. Math. Phys. 305 (2011), 427-440.

[19] F. Gabbiani and J. Fröhlich, 'Operator algebras and conformal field theory', Commun. Math. Phys. 155 (1993), 569-640.

[20] R. Haag, Local Quantum Physics: Fields, Particles, Algebras, 2nd edn, Texts and Monographs in Physics (Springer-Verlag, Berlin, 1996).

[21] G. Lechner, 'Polarization-free quantum fields and interaction', Lett. Math. Phys. 64 (2003), 137-154.

[22] G. Lechner, 'Construction of quantum field theories with factorizing $S$-matrices', Commun. Math. Phys. 277 (2008), 821-860.

[23] G. Lechner, 'Deformations of quantum field theories and integrable models', Commun. Math. Phys. 312 (2011), 265-302.

[24] G. Lechner, J. Schlemmer and Y. Tanimoto, 'On the equivalence of two deformation schemes in quantum field theory', Lett. Math. Phys. 103 (2012), 421-437.

[25] G. Lechner and C. Schützenhofer, 'Towards an operator-algebraic construction of integrable global gauge theories', Ann. Henri Poincaré 15 (4) (2014), 645-678.

[26] R. Longo, 'Real Hilbert subspaces, modular theory, $\operatorname{SL}(2, \mathbf{R})$ and CFT', in Von Neumann Algebas in Sibiu: Conference Proceedings, Theta, Bucharest, 2008, 33-91.

[27] R. Longo and E. Witten, 'An algebraic construction of boundary quantum field theory', Commun. Math. Phys. 303 (2011), 213-232.

[28] M. Müger, 'Superselection structure of massive quantum field theories in 1 + 1-dimensions', Rev. Math. Phys. 10 (1998), 1147-1170.

[29] S. N. M. Ruijsenaars, 'Scattering theory for the Federbush, massless Thirring and continuum Ising models', J. Funct. Anal. 48 (1982), 135-171.

[30] S. N. M. Ruijsenaars, 'The Wightman axioms for the fermionic Federbush model', Commun. Math. Phys. 87 (1982/83), 181-228.

[31] B. Schroer, Localization and nonperturbative local quantum physics 2012, arXiv:hep-th/9805 093.

[32] F. A. Smirnov, Form Factors in Completely Integrable Models of Quantum Field Theory. Advanced Series in Mathematical Physics, vol. 14 (World Scientific Publishing Co. Inc., River Edge, NJ, 1992).

[33] M. Takesaki, Theory of Operator Algebras. II, Encyclopaedia of Mathematical Sciences 125 (Springer-Verlag, Berlin, 2003), Operator Algebras and Non-Commutative Geometry, 6.

[34] Y. Tanimoto, 'Construction of wedge-local nets of observables through Longo-Witten endomorphisms', Commun. Math. Phys. 314 (2012), 443-469.

[35] Y. Tanimoto, 'Noninteraction of waves in two-dimensional conformal field theory', Commun. Math. Phys. 314 (2012), 419-441.

[36] M. Weiner, 'An algebraic version of Haag's theorem', Commun. Math. Phys. 305 (2011), 469-485. 